\title{
Emittance growth due to static and radiative space charge forces in an electron bunch compressor
}

\author{
Richard Talman \\ Laboratory of Elementary Particle Physics, Cornell University, Ithaca, New York 14853, USA \\ Nikolay Malitsky \\ Brookhaven National Laboratory, Upton, New York 11973, USA
}

Frank Stulle

CERN, Geneva, Switzerland

(Received 15 October 2007; published 15 January 2009)

\begin{abstract}
Evolution of short intense electron bunches passing through bunch-compressing beam lines is studied using the UAL (Unified Accelerator Libraries) string space charge formulation [R. Talman, Phys. Rev. ST Accel. Beams 7, 100701 (2004); N. Malitsky and R. Talman, in Proceedings of the 9th European Particle Accelerator Conference, Lucerne, 2004 (EPS-AG, Lucerne, 2004); R. Talman, Accelerator X-Ray Sources (Wiley-VCH, Weinheim, 2006), Chap. 13]. Three major configurations are studied, with the first most important and studied in greatest detail (because actual experimental results are available and the same results have been simulated with other codes): (i) Experimental bunch compression results were obtained at CTF-II, the CERN test facility for the "Compact Linear Collider" using electrons of about $40 \mathrm{MeV}$. Previous simulations of these results have been performed (using TRAFIC4* [A. Kabel et al., Nucl. Instrum. Methods Phys. Res., Sect. A 455, 185 (2000)] and ELEGANT [M. Borland, Argonne National Laboratory Report No. LS-287, 2000]). All three simulations are in fair agreement with the data except that the UAL simulation predicts a substantial dependence of horizontal emittance $\epsilon_{x}$ on beam width (as controlled by the lattice $\beta_{x}$ function) at the compressor location. This is consistent with the experimental observations, but inconsistent with other simulations. Excellent agreement concerning dependence of bunch energy loss on bunch length and magnetic field strength [L. Groening et al., in Proceedings of the Particle Accelerator Conference, Chicago, IL, 2001 (IEEE, New York, 2001), http://groening.home.cern/groening/csr_00.htm] confirms our understanding of the role played by coherent synchrotron radiation (CSR). (ii) A controlled comparison is made between the predictions of the UAL code and those of CSRTRACK [M. Dohlus and T. Limberg, in Proceedings of the 2004 FEL Conference, pp. 18-21, MOCOS05, available at http:// www.JACoW.org], a code with similar capabilities. For this comparison an appropriately new, $50 \mathrm{MeV}$, "standard chicane" is introduced. Unlike CSRTRACK (which neglects vertical forces) the present simulation shows substantial growth of vertical emittance. But "turning off" vertical forces in the UAL code (to match the CSRTRACK treatment) brings the two codes into excellent agreement. (iii) Results are also obtained for $5 \mathrm{GeV}$ electrons passing through a previously introduced "standard chicane" [Coherent Synchrotron Radiation, CSR Workshop, Berlin 2002, http://www.desy.de/csr] [of the sort needed for linear colliders and free electron lasers (FEL's) currently under design or construction]. Relatively little emittance growth is predicted for typical bunch parameters at such high electron energy. Results are obtained for both round beams and ribbon beams (like those actually needed in practice). Little or no excess emittance growth is found for ribbon bunches compared to round bunches of the same charge and bunch width. The UAL string space charge formulation (like TRAFIC4 and CSRTRACK) avoids the regularization step (subtracting the free-space space charge force) which is required (to remove divergence) in some methods. Also, by avoiding the need to calculate a retarded-time, four-dimensional field history, the computation time needed for realistic bunch evolution calculations is modest. Some theories of bunch dilution, because they ascribe emittance growth entirely to CSR, break down at low energy. In the present treatment, as well as CSR, all free-space Coulomb and magnetic space charge forces (but not image forces), and also the centrifugal space charge force (CSCF) are included. Charge-dependent beam steering due to CSCF, as observed recently by Beutner et al. [B. Beutner et al., in Proceedings of FEL Conference, BESSY, Berlin, Germany, 2006, MOPPH009], is also investigated.
\end{abstract}

DOI: 10.1103/PhysRevSTAB.12.014201

PACS numbers: 29.27.Bd, 29.27.Fh, 41.60.- m, 41.75.-i 


\section{INTRODUCTION}

Many modern accelerator projects depend on preserving the (small) emittances of short intense electron beams produced by linear accelerators. These projects include FEL's (free electron lasers), ERL's (energy recovery linacs), and ILC (International Linear Collider) and CLIC (Compact Linear Collider). To produce short bunches these projects employ bunch-compressing chicanes. Space charge effects in the magnets of these chicanes can degrade performance by causing emittance growth. Experiments at CERN using the CTF-II have provided substantial experimental data relevant to this issue [1-6].

In a bunch-compressing chicane the beam suffers a ,,+-+ sequence of bends, resulting in no net deflection. This causes a momentum-dependent shift $\Delta c t(\delta)$ in longitudinal coordinate $c t$ of a particle (relative to bunch center); here $\delta$ is the fractional momentum offset. The parameter quantifying this effect is $R_{56}$, typically some tens of millimeters, with $\Delta(c t)=R_{56} \delta$ (to linear approximation). The value of $R_{56}$ is governed by the bend angles in the chicane. By running the electron beam "off crest" through the linac, it is possible to produce a "chirped" beam for which $\delta$ is proportional to $c t ; \quad \delta=[d \delta / d(c t)] c t \quad$ (also to linear approximation). Working together, these two effects can convert a long bunch at the chicane input into a short bunch at the output.

The electron energies of the CTF-II electron beams are about $40 \mathrm{MeV}$. At this energy, though the electron speeds differ negligibly from $c$, the particles are not so stiff as to make free space, space charge forces negligible. The CTF-II references given above, especially [6], after discussing various influences on their observed data, conclude that coherent synchrotron radiation (CSR) forces cause the qualitatively most striking aspects of their data. (It is because there is only CSR at wavelengths greater than the bunch length that CSR becomes important only for short bunches.) While agreeing with the importance of this force, the present paper also emphasizes the importance of conventional, nonrelativistic, Coulomb forces, at the quite low energy of the data, and furthermore includes the centrifugal space charge force (CSCF) [7], which can be regarded as the transverse counterpart of CSR.

Unlike the longitudinal self-force, the transverse selfforce has a nonlinear dependence on transverse position in the bunch. This force is known to be an even function of the horizontal displacement [8] (at least for cases having the bunch long enough for the magnitude of the transverse fields to be proportional to the longitudinally local charge density). The leading CSCF effect is therefore a steering (but no focusing) of the bunch as a whole. The leading nonlinear moment is sextupole. That such a force can cause horizontal emittance growth should not be surprising; it is the magnitude of the effect that needs to be determined. Furthermore, the field shape may be more complicated for short bunches.

The string space charge model is thoroughly described in Refs. [9-11], and some recent refinements are described in the Appendix to the present paper. The main idea is that the (appropriately retarded) space charge forces simply cause intrabeam scattering. Each particle exerts an electromagnetic force on every other one. In this approach, with total energy (bunch plus radiation) being conserved, the effects of coherent radiation are implicitly incorporated by the bunch "self-work," as intrabunch forces of action and reaction do not exactly cancel.

If it were computationally possible for a simulation to use $10^{10}$ particles, nobody could take exception to this approach. But the highly singular $1 / r^{2}$ Coulomb force is usually regarded as making this approach unworkable for simulations that (because of computation time) restrict the number of macroparticles to, say, $10^{3}$. The occasional near encounters would give hopelessly erratic results if counter measures are not taken. To smooth this behavior a common approach is to take an intermediate step in which, using PIC (particle-in-cell) code, one evaluates effective fields (on a grid). In a subsequent step, deflections of individual particles are calculated by interpolation between points on the grid.

In the string approach, to suppress the close-encounter divergence, each particle is treated as a longitudinally aligned string (or needle) as regards the forces it causes. But the particle is treated as a point for purposes of determining its orbit. The dependence on (artificial) string length is suppressed by using small (but not too small) values. (The string can also be assigned a tiny height, making it a vertically aligned ribbon.) This computational approach scales poorly (time proportional to $N_{p}^{2}$, where $N_{p}$ is the number of macroparticles) but, for a single pass through a relatively short beam line, a single laptop computer provides sufficient compute power for the calculations in this paper.

Because of the extreme complication of the CSR and CSCF forces, it would greatly aid the design of the various projects mentioned earlier to distill out an empirical phenomenological parametrization of the dependence of emittance growth on the important parameters such as bunch charge, bunch dimensions, particle energy, lattice parameters, and magnetic field. We have not, as yet, obtained any such parametrization. We specialize primarily to the CTFII results.

An issue of special importance for this paper is dependence on transverse bunch dimensions. The relevant CTFII measurements concern dependence on Twiss function $\beta_{x}$ (at the chicane location) with all other parameters held 
constant. According to Ref. [6] the TRAFIC4* [12] ${ }^{1}$ and the ELEGANT [13] codes predict the emittance growth to be independent of $\beta_{x}{ }^{2}$ In Ref. [6], Braun et al. therefore register surprise, and state that they have no explanation for the strong dependence on $\beta_{x}$ that they observe. Their data is shown below in Fig. 8, along with further discussion. $^{3}$

Using energy conservation, the energy emitted in CSR can be equated to the self-work done by the bunch force acting on the bunch itself. But this force is a function of longitudinal position in the bunch. Particles at the front of the bunch are actually accelerated and particles at the back of the bunch are decelerated enough to make up the total radiated energy. The conventional wisdom has been that transverse emittance growth is dominated by the betatron excitations induced with this longitudinal dependence. The strong dependence on $\beta_{x}$ observed experimentally suggests that other factors are also important.

Another surprise noted by the authors of Ref. [6] is the absence of dependence on vacuum chamber height. This issue, important though it is, is not investigated in this paper.

\section{PROBLEMATICAL ISSUES}

\section{A. Theoretical}

Emittance growth due to space charge effects brings in at least three difficult areas of physics: relativity, radiation theory, and self-consistent multiparticle evolution. Not surprisingly, the subject has been the source of considerable controversy, with only fairly sparse experimental data available to discipline theoretical predictions.

Even when motivated by modern multi-GeV energy scale projects, what experimental data there is tends to concern short, intense, electron bunches having energies in the range from 10 to $50 \mathrm{MeV}$. This range is made

\footnotetext{
${ }^{1}$ In this paper certain theoretical simulations of CTF-II results are described as using the code TRAFIC $4^{*}$. The intention of the asterisk is to provide the following warning. The TRAFIC4* predictions come from the CTF-II papers, not directly from TRAFIC4 authors. These simulations were performed a long time ago and, like all codes, TRAFIC4 has evolved considerably in the meantime. In particular, a better modeling of the charge distributions has become possible due to the increased computer speed. The same comments apply to a lesser degree to simulations labeled ELEGANT. But then, as now, ELEGANT simply neglects all coherent transverse space charge forces.

${ }^{2} \mathrm{~A}$ basis for neglecting dependence of longitudinal force components on transverse beam dimensions is that, the characteristic ratio $\sigma_{x}^{3} /\left(R \sigma_{l}^{2}\right)$ being small compared to 1 (as it is); this assures that the transverse bunch size is small compared to the distance over which the longitudinal CSR force is roughly constant [14].

${ }^{3}$ Recent investigations of dependence on lattice function values at the compressor location have been performed by Dohlus and Limberg [15], and by Stulle [16].
}

especially difficult by the fact that the electrons are not fully relativistic. At low energies the important forces are Coulomb and Biot-Savart. But once the speeds are close to $c$ the longitudinal Coulomb force cannot much influence the bunch length. Coulomb forces can, however, increase the energy spread. Furthermore, with the order $\gamma^{2}$ cancellation of transverse electric and magnetic forces not yet being fully effective, the transverse distributions distort in ways that may or may not give irreversible transverse emittance growth. At higher energies, retardation effects and radiation become relatively more important and they also may or may not produce irreversible transverse emittance growth downstream. A brief (and we believe impartial) chronology of these investigations is contained in the paper of Li and Derbenev [17]. Particular emphasis in their paper is placed on a conjectured cancellation that is thought to influence the emittance growth. However, the abstract to a subsequent paper of Geloni, Saldin, Schneidmiller, and Yurkov [18] states that “... several existing theoretical analyses ... depend on the so-called cancellation effect. In this paper we explain why in our view such an effect is not of practical nor of theoretical interest." It may be that this controversy is semantic, though recent observations of Beutner et al. [19] exhibit strong transverse beam steering. In any case, our simulation does not require us to take either side. And, though our results would, in principle, be relevant, we make no attempt to use them to referee the controversy.

Applicable in the very high energy region, there is regularization procedure, initially due to Tamm, in which infinities in the theory are canceled by subtracting the space charge force that a charge would feel in the absence of magnetic field. As updated by Saldin et al. [20], this procedure reduces the dimensionality of the problem and permits an elegant closed form treatment of longitudinal forces. The simulation code ELEGANT [13] follows this approach. Since this is equivalent to setting all space charge forces to zero in drift regions, this procedure breaks down at low energies (where space charge forces in drift regions are obviously important). Because of this, codes like Unified Accelerator Libraries (UAL), TRAFIC4*, and CSRTRACK retain three-dimensional treatment and suppress divergence by introducing extended sources.

This paper, which is primarily numerical and phenomenological, applies formulas [10] that purport to be valid through the difficult partially relativistic region just described, as well as in the high energy region of ultimately greater interest. This calculation is very much in the spirit of an earlier theoretical formulation of Carlsten [21] and of the phenomenological comparison with experiment of Hajima [22]. (Regrettably neither of these papers was referred to in Ref. [10], but many formulas and figures are common to Carlsten's and this paper.) The common feature of these approaches is the suppression of the divergent quantities by replacing point charges by linearly ex- 
tended charges. Numerous other CSR calculations reduce divergence by employing elongated charge distributions, but Carlsten's and our approaches are strikingly (and, as far as we remember, unconsciously) similar.

Like the present paper, Carlsten [21] treated each particle as a string (uniform line charge in his terminology) for purposes of evaluating forces, but as a point for purposes of calculating its orbit. Retarded formulas agree between the two papers, though Carlsten retains only longitudinal forces and, seemingly without justification, drops the delta function contributions that come from the discontinuities at the ends of the line charge. (See the Jefimenko equation in Jackson [23].) For both longitudinal and transverse forces, the present calculation uses expressions that include the delta functions and express the integrals in closed form, routinely available, elliptic integral functions.

One reason for performing a numerical simulation (as contrasted with a thoroughly analytical calculation) is that some uncertain theoretical aspects of the phenomenon can be bypassed by the simulation. In this case the controversial "cancellation effect," mentioned above, is finessed by the simulation. To the extent such a cancellation correction is justified, it is intended to compensate for effects occurring during bunch evolution through the remainder of the beam line. When this evolution is handled numerically, with presumably accurate lattice functions, there is no need for a analytical description of the supposed cancellation.

Another difficult issue is that of magnetic end effects. Even treating the fringe fields as hard edged, the coherent force on a particle in the bunch does not turn on/off instantly as the bunch enters/leaves the magnet. Certainly the bunch lengths are always very small compared to the magnet lengths, which would suggest that end effects can simply be neglected. But retardation effects can make the bunches "appear" much longer in their self-interactions.

All magnets in our study are "long" in the sense that their bend angles are large compared to $1 / \gamma$, the synchrotron radiation cone half angle, where $\gamma$ is the usual relativistic factor. As a result, the interval over which particles influence each other strongly is short compared to the magnet length, $L$. Our bunches are "slender" in that $\sqrt{\sigma_{x} R} \ll L$, where $\sigma_{x}$ is the rms bunch width and $R$ is the bend radius. This characteristic length estimates the arc length over which a typical radially positive-displaced particle can lie in a cone of influence of a radially negative-displaced particle.

Another characteristic parameter is the "catch-up" distance $\left(24 s R^{2}\right)^{1 / 3}$ which is the longitudinal distance at which a signal emitted from a particle trailing by distance $s$ catches up with a particle ahead on the same orbit. For the CTF-II configuration and for $s=\sigma_{\mathrm{ct}}$, where $\sigma_{\mathrm{ct}}$ is the rms bunch length, this distance is large compared to $L$. This limits the longitudinal fraction of the bunch length that can interfere constructively to produce CSR.
This inequality implies the existence of a certain amount of "action at a distance" over lengths long compared to individual magnets. This might be thought to invalidate our quite local approach. A code like TRAFIC4 evaluates electromagnetic fields over intervals long compared to each magnet before calculating the forces on individual particles. Though this approach is certainly not wrong it is, in our opinion, unnecessary. Discussion follows.

Coherent radiation has been studied by Saldin et al. [24] and transient build up of transverse forces at magnet ends have been studied analytically by Geloni et al. [25] for particle bunches restricted to a one-dimensional path consisting of circles and straight lines. In their formulation they break the forces into a "radiative part" $F_{R}$ and a Coulomb part $F_{C}$, and subtract off the Coulomb part by the regularization trick mentioned above.

In the terminology of Ref. [24], Sec. 6.4, the CTF-II beam lines have long magnets, and "much longer" bunches, corresponding to the long "catch-up distances" evaluated above. In this case they find the radiative force $F_{R}$ in the magnets to be negligible, while $F_{C}$ is nonnegligible everywhere. (This is consistent with our CTFII simulation in which the longitudinal forces are comparable in bends and drifts.) In their conclusions these authors admit to "methodological questions" arising when "electrons radiate when the radiative force does not work on them and vice versa" and appeal to a Ginzburg book on electrodynamics for further justification. In our formalism the issue does not arise as there is no separation into $F_{C}$ and $F_{R}$.

Of the numerical examples in Ref. [25], the ones with parameters closest to being appropriate here are, in their Sec. V, Figs. 13(b) and 13(c). According to these figures the turn-on and the turn-off regions are short compared to the bunches themselves. Forces ascribable to retarded distances long compared to individual magnets, though not identically zero, are negligible.

Repeating a major implication of this discussion, irrespective of the magnetic field, longitudinal Coulomb forces can still alter the momentum of particles, especially near the front or back of the bunch, thereby indirectly altering the horizontal emittance measured downstream. These alterations are accompanied by actual radiation only to the extent the work done on the tail exceeds the work done on the head so that the bunch suffers net deceleration.

The preceding discussion has indicated some of the theoretical issues involved and the rationale supporting the UAL string space charge model. An unambiguous description of that model is contained in the following three sentences. Bending magnets are treated as having hard edges, with no "end effects." That is, a particle inside/ outside the magnet is treated as if it, and all other particles in the bunch, have always been inside/outside the magnet. (To speed up the calculation) a simplified force formula (Heaviside-Poincaré-Schott) is employed in all regions other than bending magnets. 
In this approximation there is neither turn-on nor turnoff of either transverse or longitudinal forces at bending magnet edges. Appropriately retarded forces from the entire past of all the particles are accounted for. What makes the approach approximate is that the past particle trajectories are somewhat deformed from the orbits the particles actually took.

A more accurate, but currently too ambitious for us, treatment would account for magnet end effects by more accurately modeling the particle trajectory histories.

\section{B. Experimental and computational}

Since it is emittance growth that is of primary concern, the calculation of emittance is clearly of importance. Regrettably it is not so easy to be certain that emittances as defined in the simulation are consistent with their determination in the experiment. For vanishing momentum spread, if the lattice is "linear," both transverse emittances are conserved. This behavior was confirmed to high accuracy in the simulation. But, for the compressor to work, an energy offset correlated with longitudinal position is required. "Chromatic" effects caused by the resulting bunch energy spread can complicate the transverse particle dynamics appreciably.

Especially in the space charge dominated regime, it is common for a beam "transverse emittance" (loosely defined) to increase briefly and then (in a judiciously designed beam line) decrease again. Such (seemingly) Liouville-violating behavior could be caused, for example, by a correlation between longitudinal and transverse bunch coordinates. The essence of bunch compression design is to keep such correlations under control so that they can be compensated downstream to achieve the intended minimal bunch dimensions.

A significant uncertainty in the present simulation has to do with this issue and the degree to which initial conditions and parameters in the simulation agree with the actual experimental initial conditions. In our simulation, after the transverse distributions are populated, the longitudinal energy chirp is applied. At this point the (energy)-sliced transverse distributions are independent of energy. An obvious initial check of our simulation is to "turn off" space charge forces by making the macrobunch charges zero and evolving the bunch to the end of the beam line. After doing this we find, in some cases, that a "vertical emittance" has increased by an amount that cannot be regarded as negligible. Note that this emittance, because it is evaluated without reference to particle energy, is not the same as the so-called "slice emittance." We call this vertical emittance growth "spurious."

Some possible explanations for spurious emittance growth are: bugs in the tracking code; dispersion at the end of the beam line which causes momentum-dependent transverse displacement (the analysis assumes that the dispersion at that point is zero and ascribes the entire value of $x$ for every particle to its betatron amplitude); and chaotic motion caused by nonlinearity of beam line elements.

We have grounds for ruling out all these possible explanations. With space charge turned off, the tracking code is relatively simple and has been in steady and reliable use for many years. The dispersion "leakage" at the emittance-determination point was always small enough to make negligible, for all particles, the contribution to transverse displacement of their momentum offsets. Finally, though the distributions observed in our simulation are not the pristine ellipsoids one might desire, the beam line nonlinearities seem to be too weak to account for the spurious growth.

We believe the dominant source of spurious vertical emittance growth to be due to lattice chromaticity. Though the dispersion is known to vanish at both input and output, the line is chromatic, meaning that the betatron phase advances from input to output depend on momentum. This skews the six-dimensional phase space so that, for example, the output vertical position depends on the particle momentum.

As mentioned above, such a correlation would not necessarily be serious in practice, because it could be removed by proper subsequent lattice design. But the correlation can affect the comparison between our simulation and the CTF-II experimental results. This is because, for reasons to be explained in the next section, and in spite of our best efforts, the lattice used in the simulation was not necessarily identical to the lattice actually used in the experiment.

Spurious growth is only significant for vertical emittance. In the worst case, the spurious emittance growth correction reduced the (unexpected) simulation-observed growth of vertical emittance, $\Delta \epsilon_{y}$, from 80 micron to 65 micron. Braun et al. [2] discuss this issue at some length. They emphasize the influence of the energy spreads (which are in the range of $3 \%$ to $6 \%$ ) especially in the vertical plane. They conclude: "Because of both the chromatic error and the asymmetry in the vertical measurements, we believe that the vertical emittance measurements are not very reliable and only include the data in the plots for completeness."

For the simulations in this paper, transverse emittance is measured by slice emittance. After having been binned on the basis of energy, the slice emittance is evaluated bin by bin. In our simulations the slice emittances are nearly independent of particle energy and they show negligible spurious emittance growth. It is only summed-over-energy emittances that exhibit spurious emittance growth, and only in the vertical plane.

Though all emittance growth results in this paper are based on slice emittances, they agree well with the results given in a preliminary version of this paper [26]. In that 
analysis only "summed-over-energy" emittances ${ }^{4}$ were calculated and we took an experimentalist's approach to the spurious emittance growth problem-we treated the spurious growth as a "background" that needed to be "subtracted off" or "renormalized." These approaches have now been made obsolete by working with slice emittances, and by the new realization that the original experimenters (for the same reasons) considered the vertical emittance measurements unreliable.

It would have been more satisfactory to repeat both experiments and simulations with more nearly identical linear lattice optics. Regrettably, since the CTF-II beam line had been dismantled, our proposition to CERN to repeat the measurements could not be fulfilled. The main uncertainty has to do with the linear optics following the chicanes. Of course the slice emittances cannot vary through this region.

These comments apply only to the CTF-II simulations; there is no issue of spurious growth concerning high energy "standard-chicane" results.

There is a another possible source of error which is opposite-i.e. harder to limit for standard chicane than for CTF-II. Occasional close-encounter collisions can lead to halo particles which contribute disproportionately to a quadratic moment such as emittance. We do not have an analytic condition which, if met, would limit the possible error from this source. But errors from this source in a simulation can be controlled by reducing macroparticle charge (at the cost of longer computation time) until further reduction has no effect. In our simulations it is similarly necessary to confirm the existence of a range of string length (an artificially imposed parameter) over which results are independent of string length. We have found these conditions more easily satisfied for CTF-II results than for standard chicane results. This may be because long range forces are relatively more influential than short range forces in the CTF-II case.

\section{Manifest data/Theory inconsistency}

Another difficult issue concerns the strong correlation between minimum bunch length in the compressor and average energy loss of the bunch as it passes through the compressor. This energy loss can only be caused by radiation, and one expects this radiation loss to be maximum for maximum compression, in which case it is produced predominantly near the end of the last magnet in the compressor. In this region the bunch length is approaching its minimum asymptotically. Braun et al.'s Eq. (1), gives the expected dependence (due to Schwinger [27]); the coherent power is proportional to

\footnotetext{
${ }^{4}$ What we call "summed-over-energy" emittances are sometimes referred to as "projected" emittances.
}

TABLE I. Self-consistency check.

\begin{tabular}{|c|c|c|c|c|}
\hline $\begin{array}{l}Q \\
\mathrm{nC}\end{array}$ & $\begin{array}{c}\sigma_{l, \min } \\
\mathrm{mm}\end{array}$ & $\begin{array}{c}\left|<\left\langle\Delta E_{e}\right\rangle_{\max }\right| \\
\mathrm{MeV}\end{array}$ & $\begin{array}{c}R_{56} \\
\mathrm{~m}\end{array}$ & $\frac{\left\langle\Delta E_{e}\right\rangle_{\max }}{Q R_{56}^{1 / 3} / \sigma_{l, \text { min }}^{4 / 3}}$ \\
\hline 5 & 0.13 & 0.7 & 0.045 & 0.026 \\
\hline 10 & 0.34 & 2.0 & 0.033 & 0.15 \\
\hline
\end{tabular}

$$
P_{\mathrm{coh}} \sim \frac{Q^{2}}{\rho^{2 / 3} \sigma_{l}^{4 / 3}},
$$

where $Q$ is the charge per bunch, $\sigma_{l}$ is the rms bunch length, and $\rho$ is the bend radius in the chicane. This radiated power produces a reduction $\left\langle\Delta E_{e}\right\rangle_{\max }$ in the average bunch energy that is maximum for minimum rms bunch length $\sigma_{l, \min }$. For substitution into proportionality (1), one can replace $\rho$ by $1 / R_{56}^{1 / 2}$ because $R_{56}$, the chicane strength defined below, is (approximately) inversely proportional to the square of the chicane magnet bend radius. Also $P_{\text {coh }}$ can be replaced by the maximum energy loss $\left|\left\langle\Delta E_{e}\right\rangle_{\max }\right|$.

An implication of Eq. (1) then, is that the ratio

$$
\frac{\left|\left\langle\Delta E_{e}\right\rangle_{\max }\right|}{Q R_{56}^{1 / 3} / \sigma_{l, \min }^{4 / 3}}
$$

should be roughly constant for the data sets under discussion. These quantities are shown in Table I for the two main CTF-II data sets. Constancy of the ratio (2) is far from satisfied. (Without being quite so explicit) Braun et al. [3] comment on this situation: "Indeed, while a linear longitudinal model describes well the measured bunch length dependence from $R_{56}$ for $5 \mathrm{nC}$, no set of initial conditions could be found that can describe the measured values for 10 nC." We, similarly, could find no initial conditions that could produce the measured $10 \mathrm{nC}, \sigma_{l}$ dependence.

Based on our understanding of the phenomena under study, it will not be possible to overcome this fundamental internal inconsistency in the experimental data being analyzed. Though ordinary space charge forces can distort the bunches they cannot change the mean energy. It seems that one of three main ingredients has to be in error: the bunch length or the bunch mean energy measurement or the CSR theory. (Beam-wall interaction, another possible contributor, is shown to be unimportant in another experiment [5].)

Experimental energy loss data that is not subject to this internal inconsistency is analyzed in Sec. IV F.

\section{SIMULATION DETAILS}

\section{A. Lattice}

A lattice description of (one variant of) most of the beam line used for the CTF-II experiments of 2001 [5] was graciously provided to us by Lars Groening, in the form of a MAD lattice file. See Fig. 1. However, a large matrix of lattices, for 18 values of $R_{56}$ and seven values of $\beta_{x}$ (at 

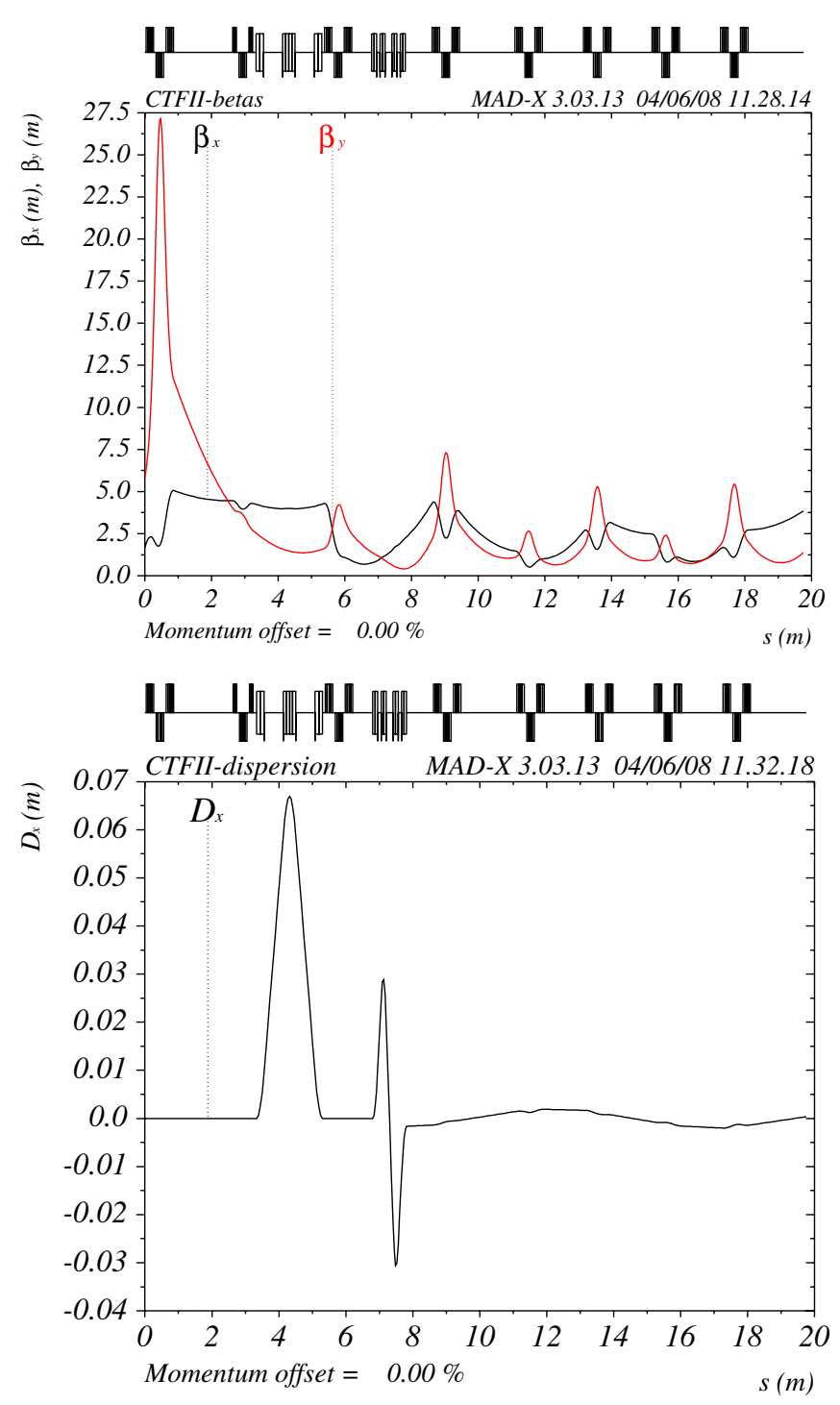

FIG. 1. (Color) Lattice functions for the CTF-II test facility (as reconstructed for $\beta_{x}=4 \mathrm{~m}$ for this report.) The lower plot shows the dispersion function with first chicane (the "compressor chicane") powered for $R_{56}=10.5 \mathrm{~mm}$ and the second chicane (the "shielded chicane") powered for $R_{56}=19 \mathrm{~mm}$. In both cases these are the values that would be obtained at the output with the other chicane turned off. The second chicane is powered off for all data except in Sect. IV F. The (slight) residual dispersion leakage in this plot is due to the shielded chicane.

chicane center), were required for this study. By design there were beam waists in both planes at the chicane center and $\beta_{y}$ was held at $1.5 \mathrm{~m}$ for most data points. Ideally, one would have used lattice description files from the original experiments. But these were unavailable because, with only one exception, the original authors had moved on from CERN and (not surprisingly) the lattice files had not been archived. Instead, for the present study, lattices were tuned up anew at each point on the matrix of parameter values. As a result, even though the lattice functions at the chicane were accurately adjusted to the correct nominal values, the lattice functions (though sensibly well behaved) were not quite identical in the simulation to those used in the actual experiment. The importance of this source of uncertainly was discussed above in Sec. II B.

Because of the large number of lattices requiring tuning, there was substantial possibility of error. In all cases, after tuning (typically six or nine of the quadrupoles at once) using MAD8, the tuned-up file was automatically translated to MAD-X format using a python script. This step is necessary because UAL requires SXF format-a fully instantiated lattice description format that can be output from MAD-X but not from MAD8. Finally the entire optics was rechecked in the UAL (finite element slice) environment. In every case the three MAD8/MAD-X/UAL lattice determinations agreed (to at least four decimal points) on all lattice functions and dispersions at the output (for identical inputs).

\section{B. Simulation procedures}

To the extent possible, electron beam parameters have been extracted from the references cited above, especially [3]. Part of the reason for concentrating on this series of experiments is their excellent planning, execution, and documentation. Nevertheless, some parameters remain unknown, such as off-crest linac phases, electron distributions, and mild deviations of the other lattice functions, $\alpha_{x}$ and $\alpha_{y}$. These parameters have had to be based on empirically fitting theory to experiment. For example, the input beam heights and angular divergences and the value of chirp factor $d \delta / d(c t)$ are fixed fairly unambiguously in this way.

The sorts of bunch distributions used are shown in Fig. 2. The number of particles per bunch was typically taken to be $N_{p}=1600$. Earlier runs with as few as 400 particles gave very similar results. In all cases, the sides of the heavy rectangles mark projected rms values of the distributions. Input parameters are listed in the caption to the figure. From the horizontal scales in the third column of this figure, one sees that the bunch has, in fact, been shortened and that the longitudinal phase space has been "overrotated." As it happens Fig. 2, with $Q=5 \mathrm{nC}, R_{56}=$ $48 \mathrm{~mm}$, is something of a "worst case," as it shows a substantial, unexpected, alteration of the vertical emittance. This feature (which cannot be inferred visually from the figure) will be discussed later.

The standard formula for beam emittance is

$$
\epsilon_{x}^{2}=\left\langle x^{2}\right\rangle\left\langle x^{\prime 2}\right\rangle-\left\langle x x^{\prime}\right\rangle^{2} .
$$

This determination of $\epsilon_{x}$ does not and, technically speaking, must not, depend on lattice functions. As a check, an alternate approach is to calculate $\epsilon_{x}$ as the rms value of the Courant-Snyder invariants of the individual particles. This determination depends on the values (presumably obtained from a linear lattice model) of the Twiss functions of the lattice at that point. From a perturbative point of view, this 

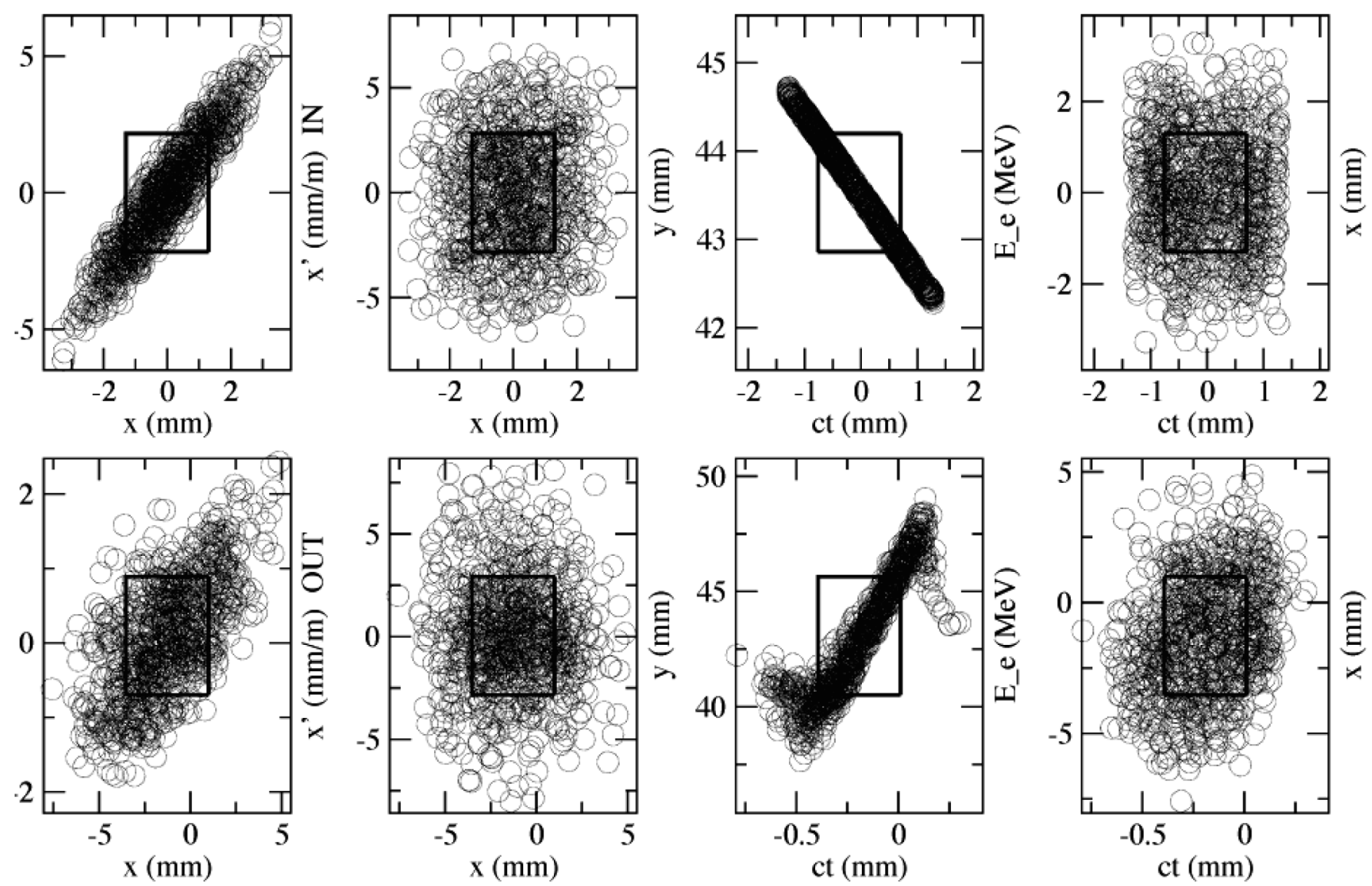

FIG. 2. Particle distributions at the entrance and exit of CTF-II for $Q=5 \mathrm{nC}, R_{56}=48 \mathrm{~mm}$. These graphs correspond to the point in Fig. 3 showing maximal (and unexpected) growth of vertical emittance. Parameters common to all these graphs are $q=5 \mathrm{nC}, N_{p}=$ $800, E_{e}=43.5 \mathrm{MeV}, l_{\mathrm{str}}=0.05 \mathrm{~mm}$. Half-widths of the initial distributions (in mm) are $\left(x_{\mathrm{hw}}, y_{\mathrm{hw}}, c t_{\mathrm{hw}}, \delta_{\mathrm{hw}}\right)=(1.3 \%, 2.7 \%$, $1.3 \%, 0.15 \%)$. Transverse distributions are Gaussian, the bunch is uniform longitudinally, with energy distribution dominated by the initial chirp. Particle locations are indicated by open circles of arbitrary (but small enough to be negligible) size. This makes individual particles visible while avoiding blackout saturation in dense regions. As it happens the circle sizes are typically comparable with the effective sizes of the macroparticles in the simulation but, to the extent this is true, it is just a coincidence.

may be a sensible approximation. In our simulations these procedures agree well.

Being based on a Monte Carlo simulation, the results in this report can depend on choice of initial seeds. Some results (such as ratios of outputs for two different $R_{56}$ values) are quite insensitive to the choice of seed. But others (such as absolute emittance growth) are sensitive to seed if the number $N_{p}$ of macroparticles is chosen too small. Apart from limiting the accuracy of the predictions, this complicates the interpretation of internal consistency checks (such as studying dependencies on number of macroparticles $N_{p}$ or string length $l_{\text {str }}$ ). Because of the strong dependence on bunch length, lumpiness in longitudinal charge distribution due to the relatively small number of macroparticles may be an important source of fluctuation. Initializing longitudinal particle coordinates on a regular grid, rather than randomly, has been found to reduce this source of fluctuation noticeably in some cases. Roughly speaking, for $N_{p}>400$ all results have become insensitive to the seed. This issue is discussed further in the Appendix. For all results given in this paper $N_{p}$ was chosen large enough to make dependence on seed negligible.

In the true physical situation a particle is subjected to an almost continuously varying space charge force. But, within the simulation, this smooth force is modeled by a vast number of tiny momentum impulses. Too-coarse slicing is a rich source of randomness which, along with too few macroparticles, provide the dominant sources of halo generation. This issue will be discussed further in connection with the standard chicane.

A complication in performing simulations like these, in which the microbunches are given spatial extension, is that an artificial parameter $l_{\text {str }}$, the string length, is introduced. It is necessary to perform studies to show that the results are insensitive to this choice. The result of choosing $l_{\text {str }}$ too short is to introduce a "halo" artifact. This limit is dis- 
cussed further in Sec. VII C. The result of choosing $l_{\text {str }}$ too long is to suppress radiative effects. This is discussed in Sec. VII. The only way to extend the range between these limits is to increase the number of macroparticles. The trade-off becomes more difficult as the bunch compression becomes more extreme. In particular, $l_{\text {str }}$ should be an order of magnitude shorter than the minimum bunch length. For the main comparisons with experiment in this paper (i.e. with the various CTF-II papers of Braun et al.), the range of acceptable values of $l_{\text {str }}$ was comfortably large. So there is little further discussion of the point. For the "standard chicane" the safe range is much shorter. Checks are described in Sec. VII.

\section{Experimental procedures and uncertainties}

The various publications from the CTF-II project describe many experimental consistency checks performed in the progress of their investigations. An especially to-besuppressed source of error would be the existence of nonvanishing dispersion at the emittance-measuring location. The chicane, made from three (or four if the center bend is
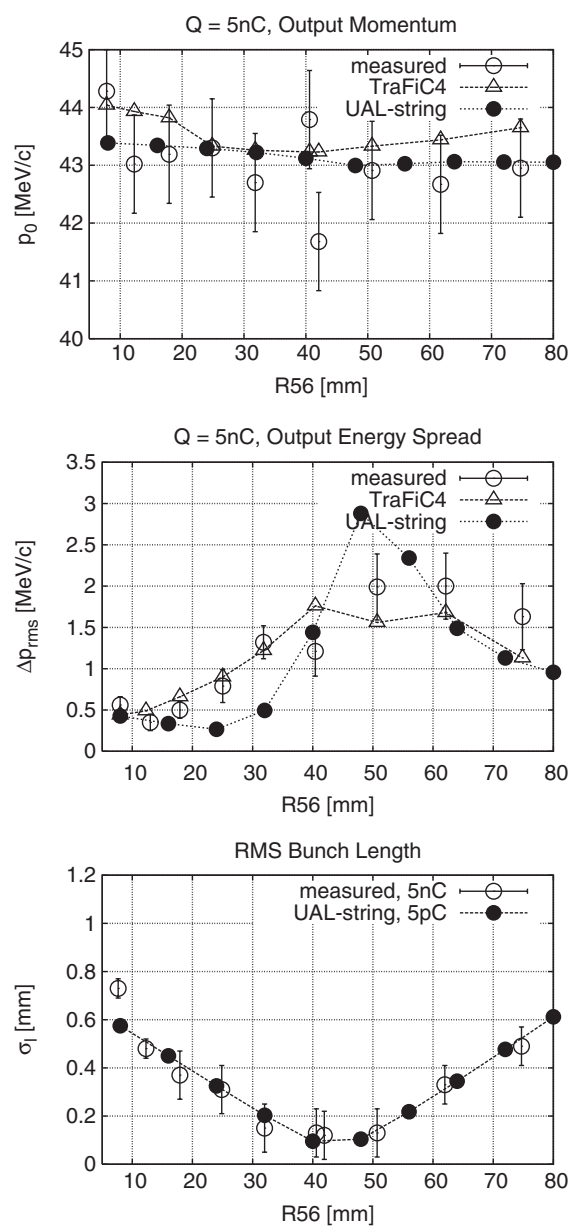

split) "rbend" magnets, in principle introduces no dispersion. This they confirmed experimentally. They do however acknowledge the presence of a longitudinally asymmetric bunch distribution and the possibility of fluctuation in the input parameters, especially to account for the less good correspondence between experiment and theory for their $Q=10 \mathrm{nC}$ data than for their $5 \mathrm{nC}$ data.

Though the issue of spurious emittance growth seems not to be emphasized in their publications, the same difficulties as the simulation has in determining transverse emittances seem likely to complicate the experimental determination. The extent to which this was investigated experimentally is unclear. It could have been done, for example, by measuring (spurious) emittance growth, for bunch charge as small as practicable, as a function of $R_{56}$. We have assumed that their linear lattice optics was tuned to be achromatic.

Another source of uncertainty concerns the initial longitudinal bunch distribution. Braun et al. describe the distribution as asymmetric and dependent upon bunch charge. In the TRAFIC4* simulations this distribution was


FIG. 3. $Q=5 \mathrm{nC}$ output momentum $p_{0}$, momentum spread $\Delta p_{\text {rms }}$, bunch length $\sigma_{l}$, and (invariant) emittances. In the bottom graphs the simulations predict a bunch length dependence on bunch charge $Q$ near the minimum. The deviations visible in these graphs suggest that the system parameters (fit empirically without accounting for this dependence) may not be quite right. The vertical emittance measurements are considered to be "not very reliable" by Braun et al. 
taken to be Gaussian. For the present paper the distribution was taken to be uniform. This choice is consistent with distributions measured in earlier experiments [28]. This is a possible source of disagreement among the data sets. Qualitatively, one expects coherent synchrotron radiation to be somewhat greater in the uniform case, than in the Gaussian case, because of the squarer edges.

\section{CTF-II AND SIMULATION RESULTS AS A FUNCTION OF VARIOUS PARAMETERS}

\section{A. Dependence on $\boldsymbol{R}_{\mathbf{5 6}}$}

In this section comparisons are made between simulations and data as functions of $R_{56}$. Brief comments are made to call attention to noteworthy features, but detailed discussion is deferred to Sec. V. The parameter choices correspond to the CTF-II data described in Ref. [3]. All data in that report are reproduced here, with the exception are shown (in Fig. 5) only for data points near bunch minimum, where maximum bunch distortion is expected. Complete sets of simulated data were taken at each of two that, for brevity, detailed output momentum distributions

values of charge per bunch $Q$, namely, $5 \mathrm{nC}$ and $10 \mathrm{nC}$. For $5 \mathrm{nC}$, dependence of output momentum $p_{0}$, and its rms spread $\Delta p_{\text {rms }}$, as well as the bunch length $\sigma_{l}$, are shown in Fig. 3. All three data sets, CTF-II, TRAFIC4*, and UAL, are in quite good agreement. Whatever disagreement there is can probably be blamed on deviant calibrations, especially of the $R_{56}$ scales, and the location of, and minimum bunch length value achieved (both of which depend on assumptions concerning the distributions of the initial electron beam).

Values of the transverse emittances are also shown in Fig. 3. In the $5 \mathrm{nC}$ horizontal case, the UAL and TRAFIC4* simulations are in quite good agreement with the data. Possibly contrary to expectation (based on the assumed CSR dominance), the vertical emittance is affected as strongly as the horizontal—but, as mentioned before, Braun et al. consider the vertical emittance measurements to be unreliable.

Figure 4 shows the same distributions for $Q=10 \mathrm{nC}$. The UAL simulation exhibits monotonically increasing (as a function of $R_{56}$ ) relative horizontal growth of about a factor of 2. In this regard there is substantial qualitative
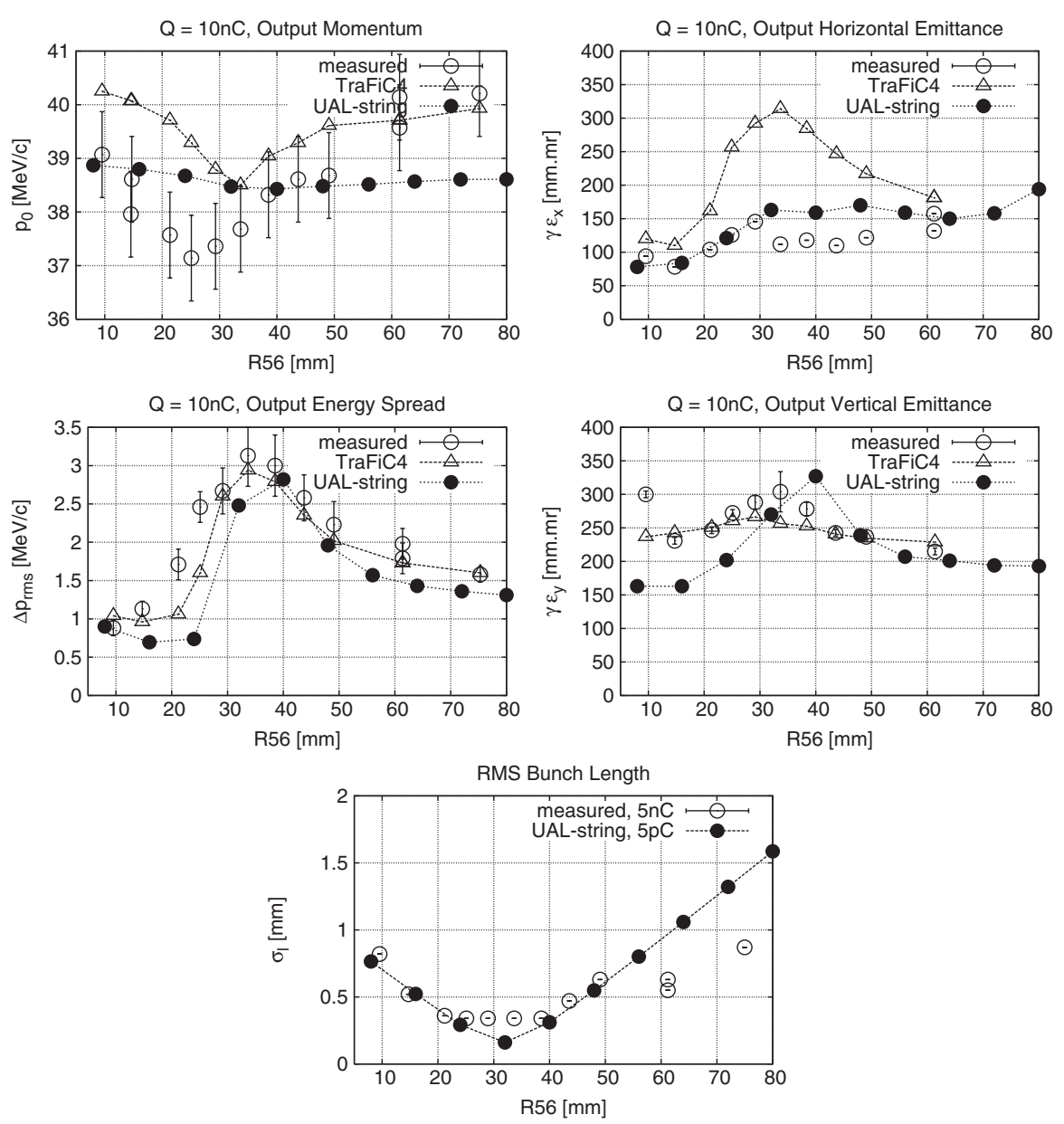

FIG. 4. $Q=10 \mathrm{nC}$ output distributions, otherwise the same as in Fig. 3. All these $Q=5 \mathrm{nC}$ and $Q=10 \mathrm{nC}$ plots used 1600 macroparticles. Results with as few as 400 particles were essentially equivalent. The vertical emittance measurements are considered to be "not very reliable" by Braun et al.. 



FIG. 5. Output energy spectra as measured, and as simulated by UAL and by TRAFIC4* for bunch charge $Q=10 \mathrm{nC}$. All three data sets are at near-maximal bunch compression with, from top to bottom, $R_{56}=34,39,44$. Distributions under the same conditions but with $Q=0$ are also shown. The so-called "double-humped" shape caused by CSR is discussed in the text.

disagreement with the TRAFIC4* simulation, which shows three-fold horizontal emittance growth, and only at intermediate values of $R_{56}$.

On the other hand, TRAFIC4* mimics well qualitatively the observed negative peak in output momentum at $R_{56} \approx$ $30 \mathrm{~mm}$ in the $10 \mathrm{nC}$ data.

Further interpretation and comment on these plots is deferred to Sec. V.

\section{B. Output momentum distributions}

Output momentum distributions are shown in Fig. 5, for the $Q=10 \mathrm{nC}$ data, in conditions just below, at, and just above bunch minimum. Measured distributions and simulation results, both UAL and TRAFIC4*, are plotted together. Also shown, for reference, are the corresponding $Q=0$ distributions. These plots clearly exhibit the energy spreading caused by space charge forces.

Some authors have commented on the "doublehumped" shape that is exhibited, especially, by the TRAFIC4* distributions. The UAL distributions would probably be better described as having a hump and a broad sloping shoulder. Considering the ragged nature of all three spectra, both simulations agree satisfactorily with the measurements. The loss of energy to radiation is presumably reflected in the spectral enhancement at the low momentum end. This shifts the bunch centroid consistent with energy having been removed from the bunch by CSR.

\section{Dependence on longitudinal position}

Dependence, on longitudinal position, of momentum spread $\sigma_{p}$ and bunch length $\sigma_{l}$ is shown in Fig. 6. In this
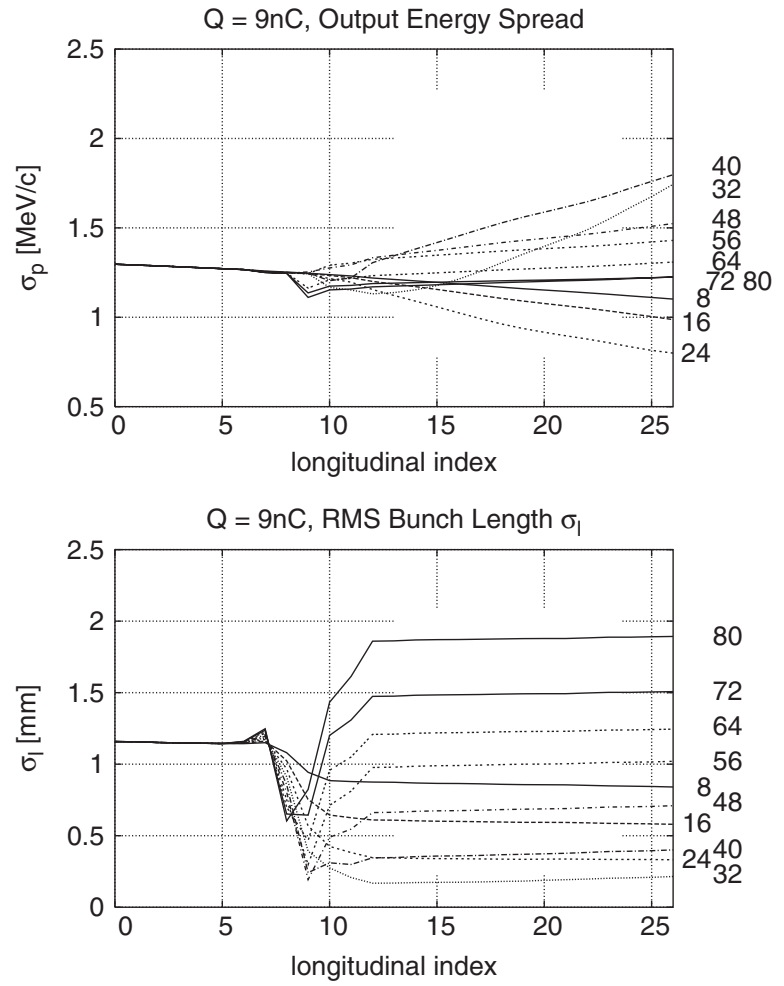

FIG. 6. Dependence on longitudinal position of momentum spread $\Delta p$ and bunch length $\sigma_{l}$. To exhibit variation within the first chicane, the horizontal scale is expanded from index location $i=6$ to $i=12$. The horizontal scale is uniform from $s=0$ at $i=0$ to $s=3.0 \mathrm{~m}$ at $i=6$ and from $s=5.1$ at $i=12$ to $s=19.8 \mathrm{~m}$ at $i=26$. Values of $R_{56}$ in millimeters are listed on the right. 
case there are no experimental data points (because there are no sensors along the beam line), but this data can be compared with Fig. 7 in Ref. [3] (not reproduced here), which shows results from the TRAFIC4* simulations. The horizontal scale of Fig. 6 is distorted in the region of the first chicane to display dependence in that region. The agreement between UAL and TRAFIC4* is excellent for this dependence. Basically the bunch shortens in the chicane and preserves its bunch length from then on. On the other hand, the energy widths grow continuously after the chicane. The growth is proportional to path length and depends on the bunch length. For the value of $R_{56}$ that minimizes $\sigma_{l}$ the growth rate of $\sigma_{p}$ is maximal.

The growth of $\sigma_{p}$ with $s$ can be explained analytically, with no need for simulation. Particles near the front of the bunch are pushed forward by the intrabunch Coulomb force within the bunch and particles near the back are pushed back. Since all particles are traveling at the same speed these forces have no effect on the bunch length; but they $d o$ affect the spread of energies. For values of $R_{56}$ below that at which the bunch length is minimized the particles near the front of the bunch have the reduced energy they (intentionally) had coming out of the linac. In this region the energy spread therefore decreases with increasing $s$. For values of $R_{56}$ above that at which the bunch length is minimized the longitudinal phase space has been over-rotated, so the particles at the front have excess energy. In this region the Coulomb force increases the energy spread, though more weakly in cases where the bunch length is long.

\section{Transverse bunch displacement}

Figure 7 shows transverse bunch displacements and slopes at the end of the beam line as predicted by the simulation. For these plots there are no experimental data as the experimenters assumed the absence of beam steering or, at least, they report no instrumentation to detect beam steering. Charge-dependent steerings like this have been observed in experiments of Beutner et al. [19]. These deflections may be caused by the centrifugal space charge force or by reduced centroid energy.

Left uncorrected (because unexpected by the experimenters) this beam steering at the input of the momentum analyzer can cause appreciable errors in the measured dependence on $R_{56}$. Adapting formulas from Chautard [28], Eq. (3.29), correction formulas for momentum errors caused by deviations $\Delta x$ and $\Delta x^{\prime}$, are

$$
\begin{aligned}
& \left.\frac{\delta p}{p}\right|_{\Delta x}=\frac{\cos \alpha}{\rho(1-\cos \alpha)+\lambda \tan \alpha} \Delta x \\
& \left.\frac{\delta p}{p}\right|_{\Delta x^{\prime}}=\frac{\rho \sin \alpha+\lambda / \cos \alpha}{\rho(1-\cos \alpha)+\lambda \tan \alpha} \Delta x^{\prime} .
\end{aligned}
$$

Here $\rho$ is the bend radius in the spectrometer, $\alpha$ is the bend angle, and $\lambda$ is the distance from the output of the analyzing magnet to the detection screen.

Neglect of these steering effects could introduce error into the measurement of the output momentum distribution. The uncertainties in momentum could be as great as $0.4 \mathrm{MeV} / c$, assuming the steering effects shown in Fig. 4, and assuming input and output spectrometers are identical. Though appreciable, this correction would not be large enough to account for disagreements shown in Fig. 4.

\section{E. Dependence on $\boldsymbol{\beta}_{x}$ at chicane center}

To investigate how emittance growth depends on transverse bunch size, the CTF-II group took data over a series of lattices that differed only in the value of $\beta_{x}$ at the center of the chicane $[5,6]$. This data, along with the UAL and TRAFIC4* and, in one case, the ELEGANT simulations, are shown in Fig. 8. To generate these graphs simulations were run, for each of several values of $\beta_{x}$, for fixed values of $R_{56}$ ranging from minimum (little bending in the chicane magnets) to maximum (large bending in the chicane magnets).
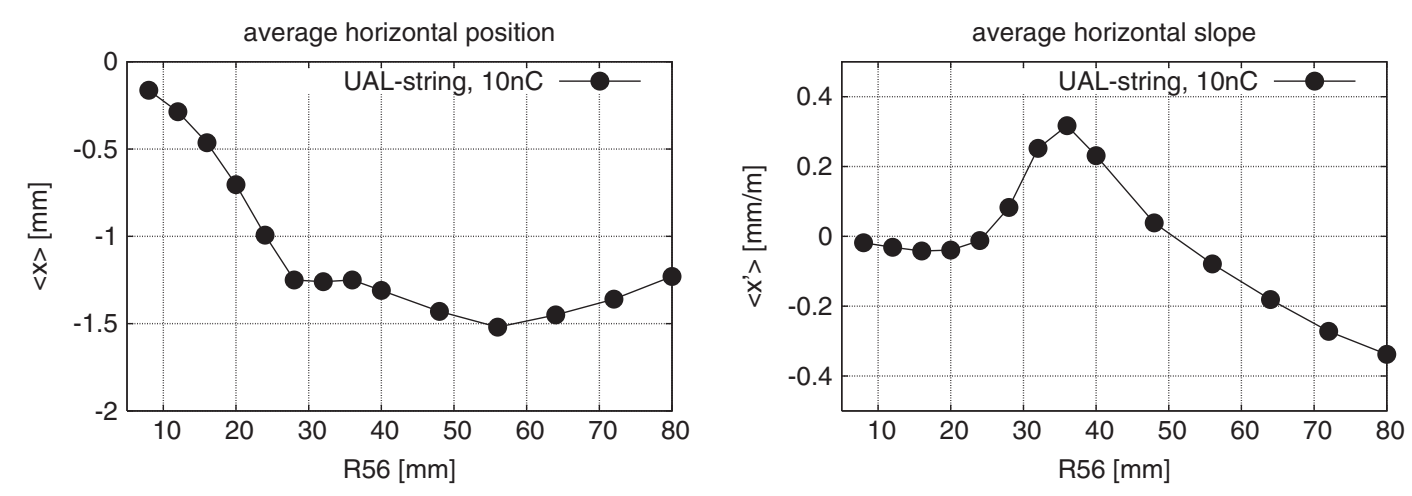

FIG. 7. Charge-dependent bunch steering for the $Q=10 \mathrm{nC}$ data set. The upper and lower graphs give, respectively, the mean bunch displacement and slope of the bunch centroid at the beam line exit. 

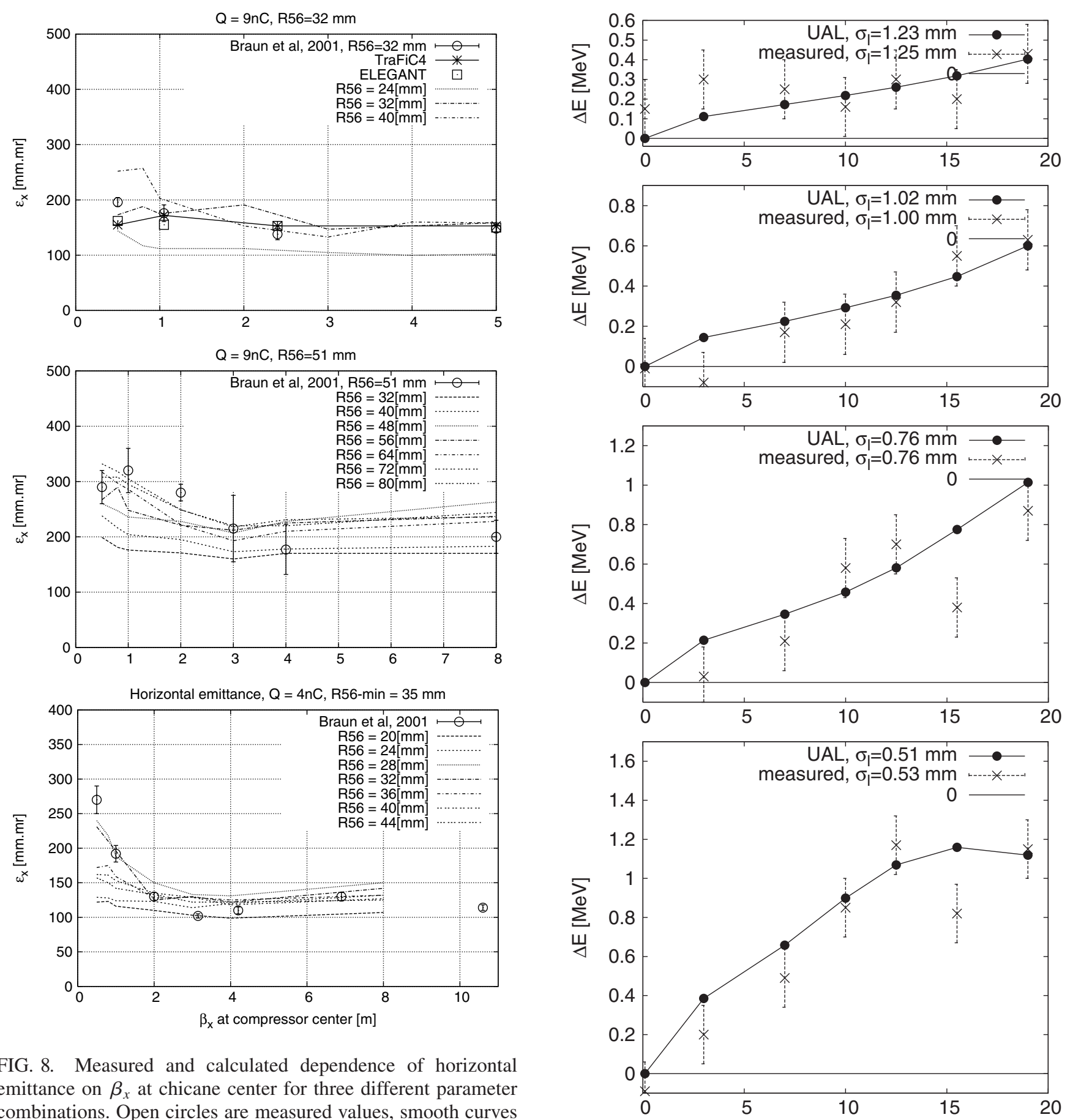

FIG. 8. Measured and calculated dependence of horizontal emittance on $\beta_{x}$ at chicane center for three different parameter combinations. Open circles are measured values, smooth curves are UAL string calculations. TRAFIC4* and ELEGANT (upper graph only) simulations predict "no significant dependence on the beta function" [6].

\section{F. Energy loss in the "shielded chicane"}

Braun et al. also performed experiments (somewhat later than those described so far, and presented at the 2001 PAC Conference [5]). These experiments were intended to emphasize accurate measurement of CSR energy loss and its possible dependence on "shielding" by the vacuum chamber. [As mentioned earlier, nothing in this paper is con-

FIG. 9. Energy loss measurements by Braun et al. [5] as functions of $R_{56}$ in the shielding chicane, for bunch charge $Q=$ $15 \mathrm{nC}$ and various values of rms bunch length $\sigma_{l}$. There are no adjustable parameters for the UAL simulations in these plots.

cerned with this (important) issue of wall shielding.] Their experiments indicated that the vacuum chamber had no effect on the measurements and that is what we assume to be the case in this paper. The energy loss data from that paper, especially its dependence on bunch length and chicane amplitude, is then directly applicable to this paper. 
The data and simulation results are shown in Fig. 9. For this data the bunch charge was $Q=15 \mathrm{nC}$. The horizontal axis expresses the shielded chicane amplitude as the value of $R_{56}$ at the output of the beam line with the compressor chicane turned off. The bunch length standard deviation varies in equal steps from $1.22 \mathrm{~mm}$ in the top graph to $0.52 \mathrm{~mm}$ in the bottom graph.

There are no adjustable parameters in these comparisons. Other than the given independent variables $R_{56}$, and $\sigma_{l}$, all other parameters are retained from the earlier analyses. Even with no fitting the chi-squared per data point of this comparison is consistent with perfect agreement between theory and experiment.

\section{DISCUSSION OF CTF-II DATA/SIMULATION COMPARISONS}

There is reasonable overall correspondence between data and simulations. Here we will emphasize areas where the agreement is only marginal.

(1) There is qualitative agreement between CTF-II data and the TRAFIC $4 *$ simulation concerning the negative peak in $p_{0}$ in the $10 \mathrm{nC}$ data of Fig. 4. For this feature, there is qualitative disagreement with the UAL simulation.

The discussion in Sec. IIC bears on this data. It was argued there that the simulation could not simultaneously match internally inconsistent data. To obtain the observed strong radiation energy loss and its rapid dependence on $R_{56}$ would require a bunch length shorter than the UAL simulation can produce by evolving a realistic initial bunch distribution. Braun et al. concur, as quoted above in Sec. II C.

One conjectures that the TRAFIC4* simulation may have accepted the measured $Q=10 \mathrm{nC}$ bunch length values at face values (which would certainly be a reasonable approach) and obtained correspondingly large CSR effects. This could plausibly account also for the erroneous three-fold horizontal emittance growth predicted by TRAFIC4* in Fig. 4. This would be consistent with the bunch having been set up on a line, i.e., neglecting energy spread, for strong compression near minimum bunch length. This would lead to too short bunches, i.e., to strong CSR.

A suspicious feature of the experimental data of Fig. 4 is the apparent beam energy increase at $R_{56}=75 \mathrm{~mm}$. Such an increase is, of course, impossible. Since the effect is only about 2 standard deviations the result is not statistically significant. But it shows that the experimental error bars have not been underestimated.

It is suggested in Sec. IV D that the presence of chargedependent beam steering could cause error in the output momentum measurement.

For all these reasons, and because the deviations between simulation and data do not exceed two sigmas, and in spite of appearances, we consider the agreement accept- able. In any case, we have found no persuasive way of improving the agreement. This is the only feature of the CTF-II data that cannot be accounted for persuasively by the UAL simulations.

(Aside based on next-section results.-The peak in energy loss near bunch minimum is very evident in the benchmark chicane results shown below. This provides credible evidence that radiative effects are most important near the end of the final chicane magnet. Since the dispersion approaches zero asymptotically through the last magnet of the chicane, both the charge density and the magnetic field stay nearly maximal for a substantial interval there. This lengthens the path over which CSR is maximal and accounts for the observed negative peak. The excellent agreement between experiment and theory concerning mean energy loss exhibited in Fig. 9 is consistent with this discussion, and with the implied strong dependence on minimum bunch length.)

(2) Experiment and simulation (both UAL and TRAFIC4*) agree on the inexorable growth of momentum spread $\sigma_{p}$ in the drift region following the chicane, with the growth rate being dependent on bunch length (and even negative for small $R_{56}$ values). See Fig. 6 . This behavior can be understood as reflecting longitudinal electrostatic Coulomb repulsion, not necessarily specific to CSR. (The standard-chicane simulations show no such energy spread increase-rather they indicate a reduction in energy spread. This difference can be ascribed to the long bendfree region following the chicane in the CTF-II case; there is no such bend-free region in the standard-chicane case.)

(3) The shrinkage of vertical emittance observed in the CTF-II experiment, Fig. 3, is curious. No such change of vertical emittance is seen in the TRAFIC4* simulation and neither CSR nor CSCF can give the vertical forces that would be needed to produce such vertical emittance changes. In the CSRTRACK code (which was not, however used for the CTF-II data), the vertical space charge force is explicitly set to zero. In any case, as discussed above in Sec. II B, Braun et al. [2] state that the vertical emittance measurements are not very reliable and are included only for completeness. So there seems to be little reason to discuss this behavior further.

(4) In the $Q=10 \mathrm{nC}$ graph giving bunch length as a function of $R_{56}$, the measured bunch lengths fall well below both simulations, for large values of $R_{56}$. Similar behavior was already observed in Fig. 7.14 of the 1996 University of Paris thesis of Frédéric Chautard [28]. This data, taken on a very similar beam line, though at higher bunch charge (in the ratio 17/10) but also at energy higher in a quite similar ratio, 62/40, shows a similar deficit at large values of $R_{56}$ and a shift of the minimum to larger values of $R_{56}$. In any case, as already mentioned more than once, the bunch length data for $Q=10 \mathrm{nC}$ is manifestly inconsistent with other data.

(5) A striking disagreement among simulations concerns the dependence on bunch width, shown in Fig. 8. These 




FIG. 10. An updated (for low electron energy) standard chicane. Comparisons between UAL and CSRTRACK simulation codes are based on this standard. Chicane parameters are given in Table II and electron beam parameters are given in Table III.

data disagree qualitatively with the absence of dependence predicted by TRAFIC4* and ELEGANT. The UAL simulation predicts increased horizontal emittance at small beam width, perhaps due to CSCF. In any case the simulation is in qualitative, one might even say semiquantitative, agreement with the measured data.

(6) Our simulation finds substantial charge-proportional beam steering. Some previous investigations seem to have neglected this effect, possibly feeding uncertainty into the determinations of bunch parameters. Substantial chargedependent beam steering has been observed experimentally by Beutner et al. [19]. Private communication from Paul Emma, as well as reporting the observation of such steering, ascribe the steering effect primarily to the excess angular deflection accompanying the reduced (due to radiation) average bunch momentum. Since the distribution of energy loss as a function of position is unclear, it is hard to estimate the importance of this effect. Some steering is also due to CSCF.

\section{UAL/CSRTRACK LOW-ENERGY CHICANE COMPARISON}

To enable controlled comparison of UAL with the CSRaware code CSRTRACK [29], the $50 \mathrm{MeV}$ benchmark chicane shown in Fig. 10 was designed. Chicane parameters are given in Table II, beam parameters are given in Table III. All distributions are Gaussian. This benchmark case was deliberately made extreme regarding CSR emis-

TABLE II. Parameter values for chicane shown in Fig. 10.

\begin{tabular}{lcc}
\hline \hline Chicane parameters & Symbol & Value, unit \\
\hline Dipole length & $L_{B}$ & $0.50 \mathrm{~m}$ \\
Drift length & $L_{D}$ & $5.00 \mathrm{~m}$ \\
Drift length & $L_{i}$ & $1.00 \mathrm{~m}$ \\
Total chicane length & $L_{\text {tot }}$ & $13.00 \mathrm{~m}$ \\
Nominal bending angle & $\alpha_{0}$ & $2.77 \mathrm{deg}$ \\
Nominal momentum compaction & $R_{56}$ & $-25.00 \mathrm{~mm}$ \\
\hline \hline
\end{tabular}

TABLE III. Electron beam parameters. The initial charge distributions are Gaussian in all six dimensions.

\begin{tabular}{lcc}
\hline \hline Electron beam parameters & Symbol & Value, unit \\
\hline Electron energy & $E_{0}$ & $50 \mathrm{MeV}$ \\
Bunch charge & $Q_{0}$ & $1.0 \mathrm{nC}$ \\
Initial rms bunch length & $\sigma_{s, i}$ & $200 \mu \mathrm{m}$ \\
Initial peak current & $I_{p, i}$ & $600 \mathrm{~A}$ \\
Uncorrelated rms energy spread & $\sigma_{E, \mathrm{rms}} / E_{0}$ & $0.01 \%$ \\
Initial energy-position correlation & $1 / E_{0} d E / d s$ & $-20.1 \mathrm{~m}^{-1}$ \\
Initial normalized emittance & $\epsilon_{n, x}$ & $1.0 \mathrm{~mm} \mathrm{mrad}$ \\
& $\epsilon_{n, y}$ & $1.0 \mathrm{~mm} \mathrm{mrad}$ \\
Twiss functions at chicane entrance & $\beta_{x, i}, \alpha_{x, i}$ & $80.41 \mathrm{~m}, 6.254$ \\
& $\beta_{y, i}, \alpha_{y, i}$ & $22.80 \mathrm{~m}, 3.110$ \\
\hline \hline
\end{tabular}

sion and emittance blowup by choosing bunch intensity that is high for the low electron energy.

To emulate CSRTRACK (which neglects vertical intrabunch forces), as one test, the UAL simulation was run with all vertical intrabunch forces suppressed. (Neglect of vertical forces is thought to be justified at high electron

TABLE IV. Simulation results for (low energy) standard chicane at $E_{e}=50 \mathrm{MeV}$.

\begin{tabular}{lcccc}
\hline \hline Quantity & Unit & CSRTRACK & UAL-NoVert & UAL-True \\
\hline$\left.\epsilon_{x, n}\right|_{\text {in }}$ & \multirow{2}{*}{$\mathrm{mm} \mathrm{mrad}$} & 1.005 & 0.982 & 0.982 \\
$\left.\epsilon_{x, n}\right|_{\text {out }}$ & & 74.1 & 72.6 & 57.2 \\
\hline$\left.\epsilon_{y, n}\right|_{\text {in }}$ & \multirow{2}{*}{$\mathrm{mm} \mathrm{mrad}$} & 1.0 & 1.04 & 1.04 \\
$\left.\epsilon_{y, n}\right|_{\text {out }}$ & & 1.0 & 1.04 & 3.50 \\
\hline$\left.\sigma_{E}\right|_{\text {in }}$ & $\%$ & 0.402 & 0.40 & 0.40 \\
$\left.\sigma_{E}\right|_{\text {out }}$ & & 0.532 & 0.56 & 0.48 \\
\hline$\left.\sigma_{l}\right|_{\text {in }}$ & \multirow{2}{*}{$\mu \mathrm{m}$} & 200.2 & 199 & 199 \\
$\left.\sigma_{l}\right|_{\text {out }}$ & & 190.3 & 191 & 184 \\
\hline \hline
\end{tabular}

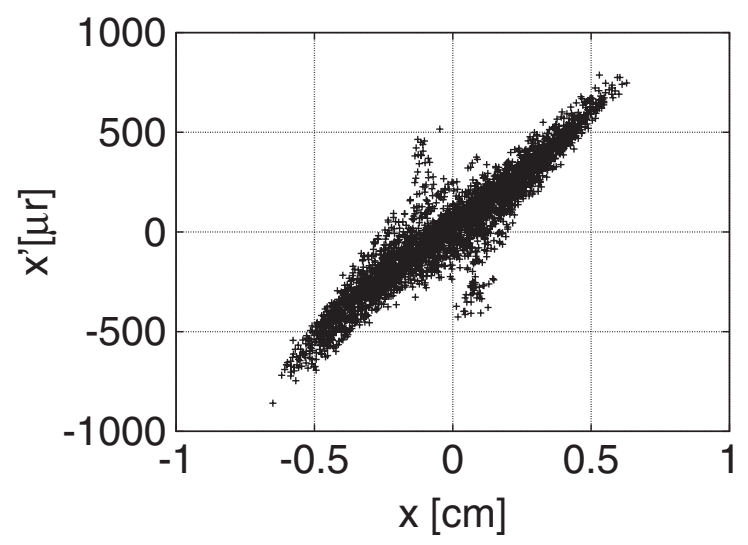

FIG. 11. Vertical-force-suppressed horizontal phase space output from UAL. The horizontal axis gives horizontal particle coordinates with the same scale as in Fig. 12. For this plot the number of macroparticles was $N_{p}=3200$. 


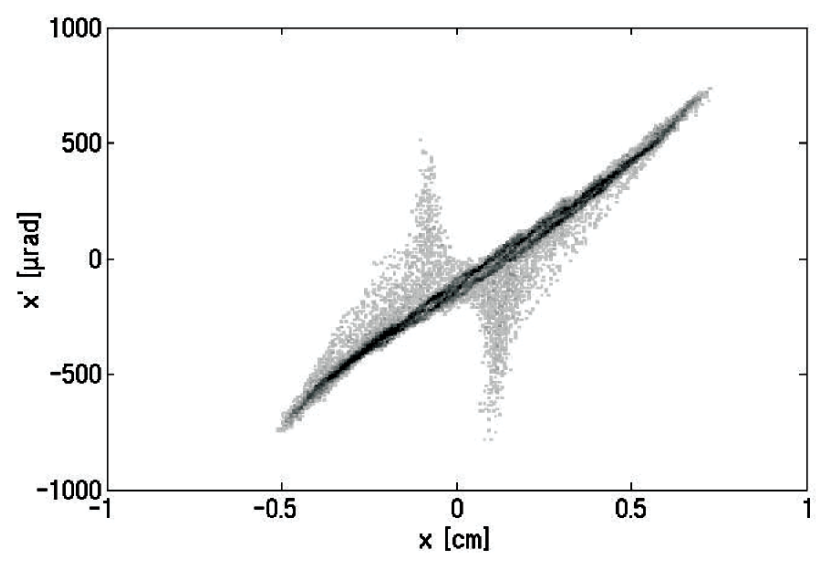

FIG. 12. Horizontal phase space output from CSRTRACK. The vertical axis is the horizontal slope, $x^{\prime}$, measured in microradians. Both scales are identical to the scales of Fig. 11.

energies since vertical and horizontal field components are relatively small at high energy. But the assumption has to break down in the limit of low electron energy.)

The third column of Table IV shows results from the CSRTRACK simulation. The fourth column shows UAL results for the case in which vertical forces are suppressed. The final column shows UAL results with all force components included - this final column gives what the UAL model considers to be the true evolution.

The bottom four rows of Table IV show excellent agreement between UAL and CSRTRACK, for energy spreads $\sigma_{E}$ and for bunch lengths $\sigma_{l}$, irrespective of the treatment of vertical forces.

Table IV also shows excellent agreement between vertical-force-suppressed UAL and CSRTRACK simulations. For the benchmark case; the output vertical emittances 74.1 and $72.6 \mathrm{~mm}$ mrad differ by $2 \%$.

For this case Figs. 11 and 12 show final horizontal phase space distributions for UAL and CSRTRACK, respectively. In both cases displacements due to residual dispersion have been subtracted. The economics of computation time limit the UAL simulation to a relatively small number of parti-

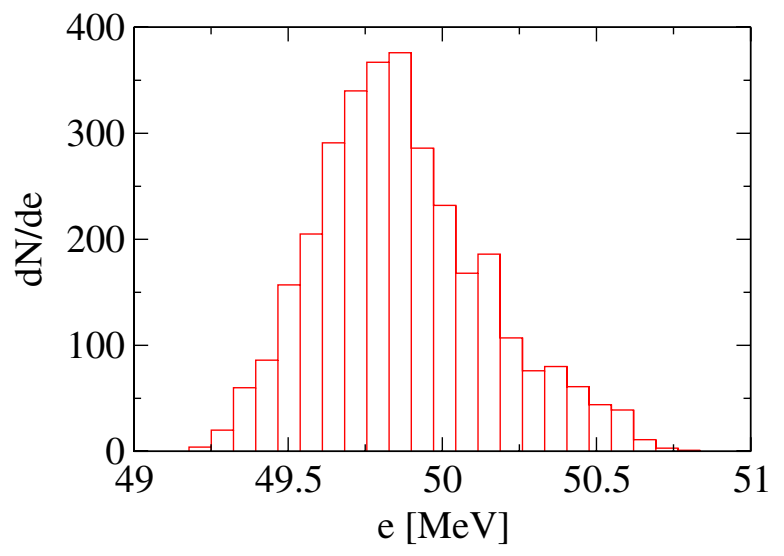

FIG. 13. UAL output energy distribution.

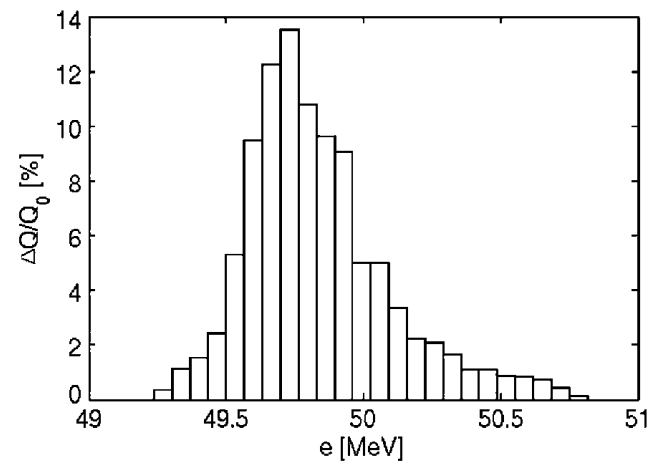

FIG. 14. CSRTRACK output energy distribution.

cles, 3200 in this case. The CSRTRACK simulation used 25000 macroparticles and 50000 test particles-the results would have been spoiled by close encounters if a smaller number of particles had been tracked. Nevertheless the plots are in good agreement. Tiny systematic shifts in both position and slope may be due to the residual dispersion subtraction.

Figures 13 and 14 show output energy spectra for the same suppressed-vertical-force case, as calculated by UAL and CSRTRACK, respectively. These also agree well.

To include the effect of vertical forces, the UAL simulation was also run with all forces, including vertical, retained. Comparing the last two columns of Table IV, the horizontal emittance increase from 57.2 to $72.6 \mathrm{~mm}$ mrad has to be regarded as an artifact of the neglect of vertical forces. Note also the substantial increase in vertical emittance, from 1.04 to $3.50 \mathrm{~mm}$ mrad, that accompanies the inclusion of vertical forces. This approximate doubling of the bunch height presumably gives a reduction in horizontal force components consistent with the reduced horizontal emittance growth.

For the comparison described in this section, the CSRTRACK computation used about $10^{6} \mathrm{CPU}$ minutes on the Swiss National Computer Center Supercomputer, while the UAL computation took about 300 minutes on one IBM T60p laptop-a 3 or 4 orders of magnitude difference. (Inclusion of vertical forces would presumably increase the ratio.) In partial compensation for the longer computation time, the far larger number of particles with CSRTRACK enables a figure such as Fig. 12 to show finer structure than Fig. 11. Within this limitation the graphs agree well. And, for calculating gross bunch properties, such as emittances, the number of particles practical for UAL simulation is sufficient.

\section{EMITTANCE GROWTH IN THE (HIGH ENERGY) "STANDARD CHICANE"}

A so-called "standard chicane," for $5 \mathrm{GeV}$ electron beams, was introduced in a 2002 workshop on coherent synchrotron radiation [30]. This was intended to enable benchmark comparisons of the various methods and computer codes. Bunch compressor emittance growth results 
TABLE V. List of benchmarked codes giving the beam parameters at the output of the chicane (from Giannessi) [31]. Results in the bottom row are from the present simulation. For the "round beam" $\gamma \epsilon_{x}=\gamma \epsilon_{y}=1 \mathrm{~mm}$ mr. For the "ribbon beam" the vertical emittance is reduced by a factor 100 . The entries marked with $*$ have been extrapolated to infinitesimal charge per macroparticle to suppress emittance growth due to halo coming from granularity of the beam in the simulation. This reduces 0.35 to 0.18 and reduces 3.26 to 0.26 . The halo is an artifact of the simulation resulting from close encounters of macroparticles in the simulation. The quite small number of halo particles has a disproportionate influence on the emittance, but would have little effect on luminosity in colliders or beam brilliance in x-ray sources. It is unclear whether a distinction between core and halo has been applied to any of the other entries in the table.

\begin{tabular}{lcccc}
\hline \hline Dimension & Code name & $\Delta E$ & $\Delta \sigma_{E}$ & $\begin{array}{c}\gamma \Delta \epsilon_{x} \\
\text { mm mr, round }\end{array}$ \\
\hline 3D & $\%$ & -0.06 & -0.002 & 0.4 \\
3D & TRAFIC4 & -0.04 & 0.017 & 1.3 \\
3D & TREDI & -0.06 & -0.006 & 0.32 \\
1D line & Li program & -0.05 & -0.004 & 0.55 \\
1D line & ELEGANT & -0.04 & -0.004 & 0.52 \\
1D line & CSR_CALC & -0.05 & -0.011 & 0.62 \\
3D & Dohlus program & -0.03 & -0.005 & $(0.18)^{*}$ \\
\hline \hline
\end{tabular}

are reviewed by Giannessi [31]. A selection of his results most relevant to the current paper are tabulated in Table V, which is copied from the original, but with a row added at the bottom and a column on the right, giving the results of the "UAL stringsc" code. In all cases (except for the final column) standard parameter values are used, as given, for example, by Emma [32]. The table shows "marginal" agreement among the various determinations for the nominal (i.e. round beam) parameters.

The entry in the final column will be considered especially here; this entry comes from reducing the vertical emittance by 2 orders of magnitude from its nominal value. (Properties of such ribbon beams are of more than academic interest, since most applications of low emittance


FIG. 15. Particle distributions at the entrance and exit from the "standard-chicane" benchmark [32]. However, to make the halo visible (in the bottom row, third plot over) the vertical emittance $\gamma \psi$ has been reduced to $0.01 \mathrm{~mm} \mathrm{mr}$ from its nominal value of $1 \mathrm{~mm}$ mr. Parameters for these graphs are $q=1 \mathrm{nC}, N_{p}=800, E_{e}=5.0 \mathrm{GeV}, l_{\text {str }}=0.3 \mu \mathrm{mmm}$. Half-widths of the initial distributions (in microns) are $\left(x_{\mathrm{hw}}, y_{\mathrm{hw}}, c t_{\mathrm{hw}}, \delta_{\mathrm{hw}}\right)=(63 \%, 3.6 \%, 200 \%, 0.0002 \%)$. 

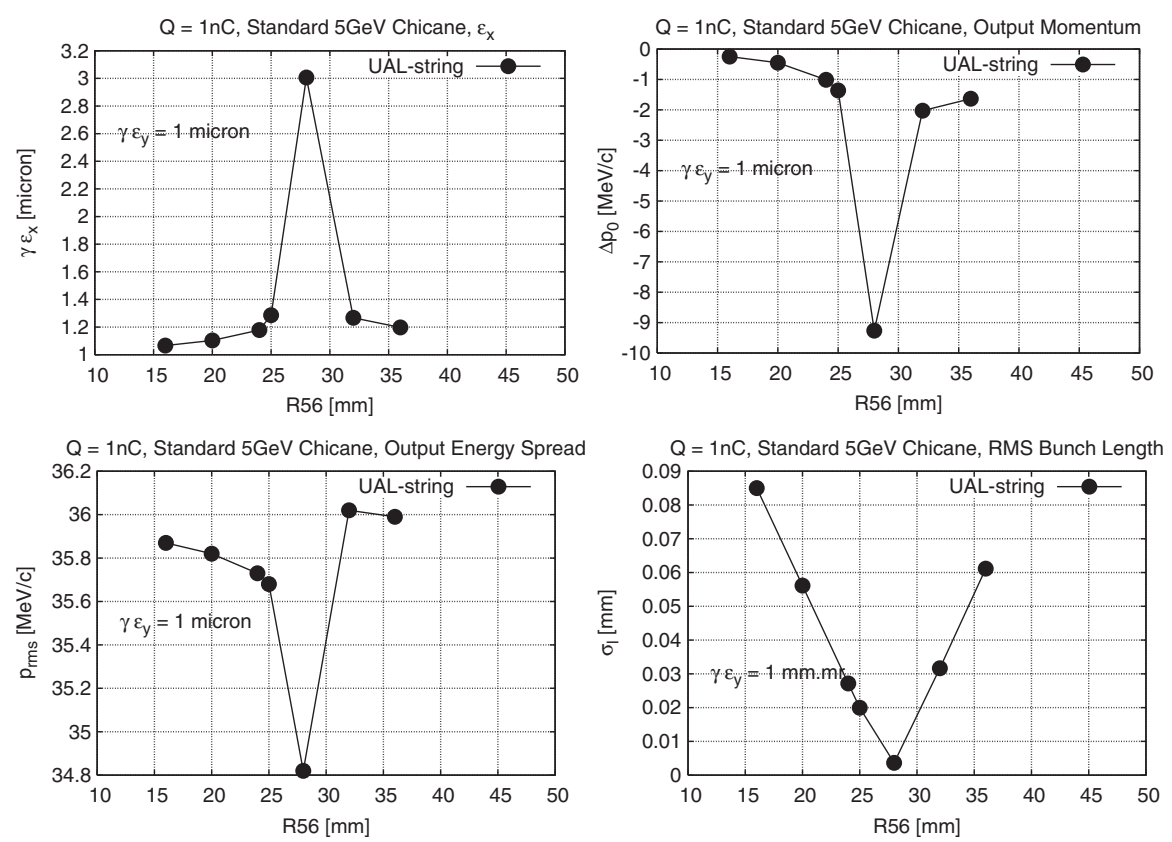

FIG. 16. Dependence on $R_{56}$ of various beam properties. All plots are for the nominal, $\gamma \epsilon_{y}=1 \mathrm{~mm} \mathrm{mr}$, round beam case, the electron bunch shape was Gaussian gridded, the string length was $l_{\text {str }}=3$ micron, $N_{p}=800$, and $n_{\text {split }}=4$.

beams require $\epsilon_{y} \approx \epsilon_{x} / 100$.) In this revised configuration the interpretation of the simulation results cannot be separated from the discussion of "halo generation" and on the dependence on the string length and string height of the string space charge model.

Figure 15 shows phase space distributions of the particles at input to and output from the compressor. Qualitative differences between standard-chicane and CTF-II behavior can be inferred by close comparison of this figure with Fig. 2-here, for example, the longitudinal distribution is Gaussian and gridded, not uniform and random. Like the earlier figure, this figure is also a "worst case"; here because the beam height has been reduced by a factor of 10 to produce a ribbon beam. This permits the pictorial exhibition of the halo production that is an artifact of the simulation. Such halo generation could similarly result from true bunch granularity and would be exhibited by any simulation code using macroparticles.

Comments about these data can be based on the graphs in Fig. 16. Especially noteworthy is the fact that a relatively small upward shift (from $R_{56}=25 \mathrm{~mm}$ to $R_{56}=28 \mathrm{~mm}$ ) produces close to an order of magnitude reduction in bunch length. ${ }^{5}$ This is not too surprising; presumably the nominal chicane parameters have been adjusted to be just below the point where bunch head and tail can change places. But nearness to this "Jacobian singularity," at the point where the bunch length is minimum, probably contributes to the

\footnotetext{
${ }^{5}$ Incidentally, the fact that the bunch length for the $R_{56}=$ $28 \mathrm{~mm}$ case is not large compared to the string length, makes the computed results unreliable in that case.
}

scatter of calculations like those in Table V. (In actual practice the uncorrelated energy spread might be larger than is assumed in the nominal benchmark parameter set; this would cause the bunch length minimum to be less sharp.) The second graph from the top shows a substantial mean energy shift at the nominal $R_{56}=25 \mathrm{~mm}$ setting (though the fractional shift is not large even at that point.) This shift is independent of the vertical emittance. This is as expected - the shift is due to CSR, which is essentially independent of transverse bunch dimensions. It will be seen shortly that "halo particles" are contributing substantially to the emittance growth shown in Fig. 16, but halo particles have little effect on the other plots.

\section{A. Dependence on string parameters and on beam aspect ratio}

In principle, the result of the simulation should be independent of number of macroparticles $N_{p}$, number of intervals $n_{\text {split }}$ into which the bends are segmented, and of the string dimensions $l_{\text {str }}$ and $h_{\text {str }}$, with the latter two values having been reduced systematically until independence is achieved. All calculated quantities have flattened out for values of $N_{p}$ exceeding 400 or so. All longitudinal quantities (or rather, all but horizontal emittance) were found also to be independent of the string length, for string lengths ranging from $0.1 \sigma_{l}$ to $0.3 \sigma_{l}$. In this range any reduction of emittance growth due to artificial bunch lengthening from the string length is negligible. See Table VI, which collects data from a few computer runs and gives their statistical fluctuations. For entries in this table $Q=1 \mathrm{nC}, R_{56}=25 \mathrm{~mm}$, with nominal, round beam 
TABLE VI. Output bunch parameters for the standard chicane with nominal parameters. All beam measures except $\epsilon_{x}$ are essentially independent of all variables exhibited. The parenthesized entries in the second column indicate the number of independent computer runs performed to obtain the remaining entries. Column heading $y h W$ stands for bunch half-height.

\begin{tabular}{lccccccccc}
\hline \hline $\begin{array}{l}y h W \\
\text { micron }\end{array}$ & $\begin{array}{c}l_{\text {str }} \\
\text { micron }\end{array}$ & $N_{p}$ & $n_{\text {split }}$ & $1 /\left(n_{p} n_{\text {split }}\right) \times 10^{-3}$ & $\begin{array}{c}\delta E \\
\mathrm{MeV}\end{array}$ & $\begin{array}{c}\delta \sigma_{E} \\
\mathrm{MeV}\end{array}$ & $\begin{array}{c}\gamma \epsilon_{x}(\mathrm{raw}) \\
\mathrm{mm} \mathrm{mr}\end{array}$ & $\begin{array}{c}\gamma \epsilon_{x 0} \\
\mathrm{~mm} \mathrm{mr}\end{array}$ & $\begin{array}{c}\gamma\left(\epsilon_{x}-\epsilon_{x 0}\right) \\
\mathrm{mm} \mathrm{mr}\end{array}$ \\
\hline 36 & $3(4)$ & 800 & 8 & 0.156 & $-1.386 \pm 0.009$ & $-0.2476 \pm 0.0037$ & 1.165 & 0.981 & $0.183 \pm 0.019$ \\
& $3(4)$ & 800 & 4 & 0.313 & $-1.377 \pm 0.002$ & $-0.2529 \pm 0.0020$ & 1.145 & 0.967 & $0.177 \pm 0.059$ \\
& $3(4)$ & 800 & 2 & 0.625 & $-1.368 \pm 0.018$ & $-0.2488 \pm 0.0090$ & 1.333 & 0.982 & $0.351 \pm 0.155$ \\
\hline 3.6 & $3(4)$ & 800 & 16 & 0.0781 & $-1.40 \pm 0.018$ & $-0.243 \pm 0.002$ & 1.330 & 0.982 & $0.348 \pm 0.087$ \\
& $3(4)$ & 800 & 8 & 0.156 & $-1.39 \pm 0.008$ & $-0.234 \pm 0.010$ & 1.372 & 0.996 & $0.441 \pm 0.101$ \\
& $3(2)$ & 1600 & 4 & 0.156 & $-1.43 \pm 0.014$ & $-0.244 \pm 0.015$ & 1.568 & 0.999 & $0.507 \pm 0.088$ \\
& $3(4)$ & 800 & 4 & 0.313 & $-1.40 \pm 0.016$ & $-0.226 \pm 0.013$ & 1.671 & 0.996 & $0.692 \pm 0.114$ \\
& $3(4)$ & 800 & 2 & 0.625 & $-1.39 \pm 0.059$ & $-0.223 \pm 0.030$ & 3.184 & 0.982 & $2.201 \pm 1.180$ \\
\hline 3.6 & $4(2)$ & 800 & 16 & 0.0781 & $-1.38 \pm 0.01$ & $-0.239 \pm 0.012$ & 1.184 & 0.996 & $0.198 \pm 0.013$ \\
\hline \hline
\end{tabular}

height 36 microns and ribbon beam vertical height $3.6 \mathrm{mi}-$ crons. Numbers in parentheses in the second column give the number of independent runs that have been combined to calculate the entries in that row.

It was found, however, especially for short string lengths, that the horizontal emittance increase is much greater for the ribbon beam than for the round beam. This behavior is exhibited in Fig. 17 which shows, in the ribbon beam case, the dependence of $\epsilon_{x}$ on the factor $1 /\left(N_{p} n_{\text {split }}\right)$ for a few values of $l_{\text {str. }}$. The $1 /\left(N_{p} n_{\text {split }}\right) \rightarrow 0$ limit of the horizontal scale is equivalent to the macrobunch charge being zero, which eliminates the production of halo particles by single close-encounter scatters. Naturally, the numerical accuracy suffers in this extrapo-

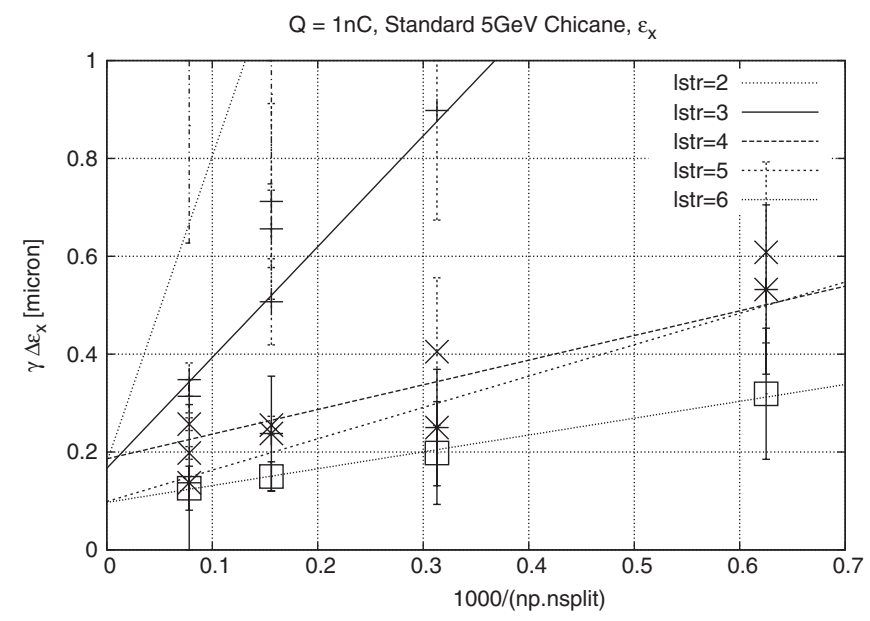

FIG. 17. Dependence on $1 / N_{p} / n_{\text {split }}$ of the measured horizontal emittance growth $\gamma \Delta \epsilon_{x}$, for various values of string length $l_{\text {str }}$, for a ribbon beam, with $\gamma \epsilon_{y}=0.01 \mathrm{~mm} \mathrm{mr}$. For these simulations the string height $h_{\text {str }}=y h W / 10=0.36$ micron. Extrapolation to the origin (infinitesimal charge per bunch) eliminates close-encounter halo production. Some residual dependence on $l_{\text {str }}$ cannot be excluded but, in any case, it can be seen (comparing with Table V) that reducing the beam height has caused no appreciable increase in horizontal emittance growth. lation, but the extrapolated values are consistent with being independent of $l_{\text {str }}$. Incidentally, it was not considered necessary to obtain greater than the $\pm 30 \%$ accuracy of the extrapolated-to-zero $\epsilon_{x}$ value shown in Fig. 17 since this data applies to the artificial, factor-of-ten-enhanced charge density, ribbon beam case.

\section{B. Interpretation of benchmark chicane results}

Using Poynting vector methods, Schwinger [27] has calculated the properties of both incoherent and coherent synchrotron radiation. Most of the properties of the radiation per se have been amply confirmed by subsequent experiments. In Schwinger's calculation the electron bunches are taken to be needle shaped, with charge distributed uniformly along their length, and with vanishing transverse dimensions. Since CSR is strongly dependent on the longitudinal bunch distribution, Schwinger's formulas cannot be applied directly to the Gaussian longitudinal bunch shape specified for the standard chicane.

The total energy radiated from an electron bunch, both coherent and incoherent, is uncontroversial, and the dependence of the longitudinal self-force (wake field) on longitudinal position is also quite well understood. For example, the longitudinal force is essentially independent of transverse position in the bunch for the needle-shaped bunches of current interest. This is the force which does the selfwork matching the radiated energy. One effect of this selfforce is to extract energy from or inject energy to the electrons, depending on their longitudinal position-this dependence gets superimposed on the preexisting energy "chirp." Because of local dispersion at the point of emission, this change of energy is immediately reflected in a changed horizontal betatron amplitude, and therefore a changed horizontal emittance.

Part of present-day intense-electron-bunch lore is that, at high electron energy, CSR is the primary source of emittance growth of an electron bunch in a magnetic field. This paper confirms this-provided that high energy means well into the $\mathrm{GeV}$ range. The theoretical basis for this lore is 
that the leading mechanism is CSR which, occurring at a point of nonvanishing dispersion, is reflected in alteration of the transverse beam dimensions downstream, even at points of vanishing dispersion.

Where our $5 \mathrm{GeV}$ simulation finds growth inconsistent with this picture the growth is ascribable mainly to a halo that is an artifact of the numerical simulation; the halo is caused by the granularity of the beam representation. If the electron beam were, in fact, granular on the same scale as the simulation (that is, on a scale of roughly the bunch dimensions divided by 10), then a halo much like that in the simulation could be produced. In any case, since this emittance increase is due to only a small fraction of the particles, this would not represent growth of the core of the beam. The luminosity (in the case of colliding linacs) or the brilliance (in the case of x-ray sources) would therefore not be much affected by such a halo.

Based on the present simulation, for the standard chicane at high energy such as $5 \mathrm{GeV}$, the effects just mentioned seem to have only small effects on transverse emittance growth compared to the CSR effect. For that matter, even the CSR-induced emittance growth is predicted to be fractionally small compared to the initial emittance. It is even suggested that some emittance growth results shown in Table $\mathrm{V}$, by neglecting the halo generation accompanying their relatively small number of macroparticles, may have noticeably overestimated horizontal emittance growth of the bunch core.

\section{Speculations concerning halo generation}

The Touschek effect, analyzed, for example, by Piwinski [33], is a well-known phenomenon that is important for $\mathrm{GeV}$-scale dense electron bunches. The Touschek lifetime in storage rings is typically of order an hour in storage rings. This suggests the effect will be negligible in a bunch compressor, but there are factors which may modify this. For one thing, the "healing" time constant for an electron suffering an encounter not quite close enough to be ejected from the storage ring is of order milliseconds rather than hours. The definition of what constitutes halo will therefore be very different for bunch compressors than it is for the Tousckek effect. A typical fractional momentum loss leading to particle loss in a storage ring might be $\Delta \delta_{\text {typ }}^{\text {Touschek }} \approx$ $10^{-2}$. A significant fractional momentum change in the bunch compressor might be

$$
\Delta \delta_{\text {signif }}^{\text {compressor }} \approx \sqrt{\frac{\gamma \epsilon_{x}}{\gamma} \frac{\beta_{x}}{D^{2}}} \approx 10^{-4} .
$$

Also, though the bunch compressor charge per bunch may not be large by storage ring standards, the bunch volume may be smaller by a factor of order $10^{4}$. As it happens, Piwinski's formulas for the Touschek effect are not directly applicable. The compressor setup depends on the correlation between momentum and longitudinal position. As the bunch approaches the end of the chicane the head and tale approach each other transversely (in the bunch's frame) as they come into coincidence longitudinally. This configuration greatly enhances the Touschek-like scattering rate. Though Piwinski's treatment assumes no such correlation, his formulation is sufficiently general to make the inclusion seem straightforward.

Rough evaluations of Piwinski's formulas suggest that, in spite of all these factors, because the operative time is so short, single close-encounter electron-electron scatters will still contribute negligibly to emittance growth within the compressor. (The same would not be true for the same bunches circulating in a ring.) In any case it is more appropriate to calculate true Touschek halo generation analytically rather than by numerical particle tracking simulation. In the present string space charge simulation a certain amount of halo generation has certainly been observed; for example, a halo of a few particles can be seen in Fig. 15. But to extract meaningful halo production rate for electrons (as contrasted with macroparticles) from the numerical simulation will require a stochastic analysis far more sophisticated than we have at present.

In the present simulation with, say 1000 macroparticles, each macroparticle has effective transverse size equal to about one-tenth of the smaller transverse bunch dimension. Bunch substructure on this scale, for example, because of gun cathode nonuniformity, may occur in practice. The simulation suggests that bunch substructure on this (or shorter) scale is likely to lead to substantial halo production.

It has been noted previously that the longitudinal parameters (mean and rms energy loss) calculated in our simulation agree well with other calculations, but that an appreciable fraction of its emittance growth is what we are now ascribing to halo. After suppressing this the emittance growth of the core predicted by our simulation is somewhat less than the growth predictions of other codes. These points may be mainly of academic interest since, for predicting the performance for future facilities, the emittance growth of the core is relatively small, even in the ribbon beam case.

There is one way in which the numerical simulation can be expected to give a faithful representation of halo behavior for real electron beams. It concerns the round/ ribbon beam comparison. Once a prescription has been established for segregating halo from core, it seems that the contribution of halo to emittance, with bunch charge held constant, will vary inversely with the bunch height, irrespective of whether real electrons or macroparticles are at issue. This inverse scaling behavior is what is observed for macroparticles in the simulation.

\section{ACKNOWLEDGMENTS}

This report has profited greatly from the careful reading of earlier versions and detailed comments made by Lars 


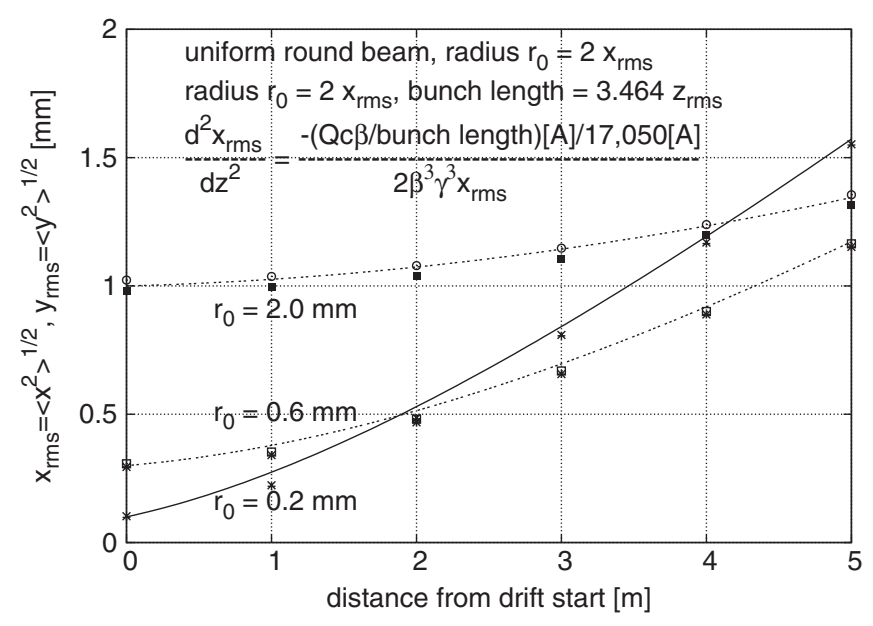

FIG. 18. Evolution of a $Q=77 \mathrm{pC}, 10 \mathrm{MeV}$ electron bunch through a drift region. Though the bunch is uniformly distributed in all three dimensions (beer can shape), its transverse dimensions are expressed as rms values of $x$ and $y$, which almost superimpose since the beam is round. The rms bunch length is $0.9 \mathrm{~mm}$. With all particles initially parallel to the axis, the smooth curves are solutions of the "envelope equation" shown.

Groening. We also thank Ivan Bazarov for insisting that our simulation successfully pass the test shown in Fig. 18. Frank Stulle would like to thank the Paul Scherrer Institut and the Swiss National Supercomputing Center (CSCS) for providing access to the Cray XT3 supercomputing facility.

\section{APPENDIX: UAL IMPLEMENTATION DETAILS}

Concerning the physics of the UAL string space charge simulation, there have been three alterations of the code (primarily influencing transverse rather than longitudinal evolution) compared to the descriptions in the references given earlier.

(To speed up the code) the forces in field-free regions are calculated analytically, using the (once-integrated) Heaviside-Poincaré-Schott formula [11] for the forces between parallel-traveling particles. This portion of the code was benchmarked against the so-called "envelope equation," which is shown in Fig. 18. This equation was solved both analytically and numerically to produce the smooth curves shown. The pairs of data points shown are simulation outputs for $x_{\text {rms }}$ and $y_{\text {rms }}$. The agreement is good. It is important for the drift sections to be sufficiently subdivided (much like the subdivision used in the numerical solution of the envelope equation) to improve precision.

For purposes of calculating the force field produced by a string, the string is also assigned a vertical height $2 y_{0}$. By choosing this height small compared to the bunch height (typically $y_{0} \approx \sigma_{y} / 10$ ), the possibility of horizontal emittance growth due to vertical bunch nonuniformity is preserved. At the same time, even the only logarithmically divergent but unphysically large (because of amplification proportional to macrobunch charge) deflections occurring in the occasional close encounter of microbunches, are eliminated. In the original paper closed form analytical formulas are given for the fields due to such extended sheets. But those formulas are very complicated and, in the UAL code, the effect of vertical string extension is modeled more crudely, but far more simply. The formulas for each of the force components acting on one string due to another are proportional to factors of the form $\left(a^{2}\right)^{-3 / 2}$, where $a$ is approximately the separation distance of the source string and the string being tracked. In a typical simulation the inequality $y_{0}^{2} \ll a^{2}$ is respected for all but a few (extremely close) encounters. Assigning vertical height to one of the strings amounts to making the replacement of this factor by its average value:

$$
\begin{aligned}
\frac{1}{\left(a^{2}\right)^{3 / 2}} & \rightarrow\left\langle\frac{1}{\left(\tilde{y}^{2}+a^{2}\right)^{3 / 2}}\right\rangle=\frac{1}{y_{0}} \int_{0}^{y_{0}} \frac{d \tilde{y}}{\left(\tilde{y}^{2}+a^{2}\right)^{3 / 2}} \\
& =\frac{1}{a_{0}^{2}} \frac{1}{\left(y_{0}^{2}+a^{2}\right)^{1 / 2}}<\frac{1}{\left(y_{0}^{2}+a^{2}\right)^{3 / 2}} .
\end{aligned}
$$

In the UAL code the initial expression in this chain of equalities and inequalities is simply replaced by the final expression. Apart from this change, all other string-model formulas are unchanged. This amounts to replacing the string by a vertical ribbon. For the vast majority of string encounters this replacement has a negligible effect, but the transverse impulse suffered in the occasional close encounter is limited. Furthermore, the longitudinal impulses should not be much affected, since they are known to be insensitive to transverse position. For the occasional close encounter, instead of potentially being uncontrollably large, the transverse deflection is limited. This procedure does, however, bring in another ad hoc parameter $h_{\text {str }}$, whose effect needs to be investigated in the simulation.

The sickness that is treated by this trick has to do with occasional an "halo particle" produced in close encounters. By varying $h_{\text {str }}$ one can investigate the prevalence and importance of the halo particles.

For the relatively small number of macroparticles for which the string space charge simulation is practical, there is another source of fluctuating dependence on Monte Carlo seed. Since emittance growth is a kind of diffusion, it can be expected to be most important in regions where the distribution functions are changing most rapidly. Distributions of a small number of macroparticles are sure to be "lumpier" than the distributions of the vastly more numerous true particles. In an attempt to cancel low order moments of the distributions, the UAL software allows the distributions to be "symmetrized." For example, a distribution of 800 particles is generated by first choosing 400 particles into nominally correct distributions and then completing the distribution with the same particles, but with their transverse phase space coordinates reversed. This trick seems to reduce fluctuations noticeably. 
As has been explained previously the maximum possible impulse imparted to a macroparticle is proportional to both the macroparticle charge and to the interval being represented numerically. Because the computation time scales only linearly with the bend splitting factor $n_{\text {split }}$, but quadratically with number of macroparticles $N_{p}$, it is useful for the UAL code to support bend splitting in its string space charge code without the need for generating a separate input lattice description for each value of $n_{\text {split }}$. For different reasons (the faithful treatment of the lattice optics), it is appropriate to similarly split quadrupoles into multiple segments. But the space charge effects in quadrupoles are quite unimportant, so the quadrupole splitting is ignored for space charge calculations. Bends are opposite-their splitting has little effect on the optics, but affects space charge. For these reasons, the UAL code supports the splitting of sbend elements for purposes of space charge calculations. (Of course such splitting can always be done in the external lattice file but the UAL code permits this to be done under the control of the ".apdf" file.)

The computer engineering updating of the code has been substantial. The actual source code can be viewed at Ref. [34]. To incorporate space charge the preexisting UAL environment needed to be augmented by only a few classes, all shown in the following ".apdf" (accelerator propagator description format) file:

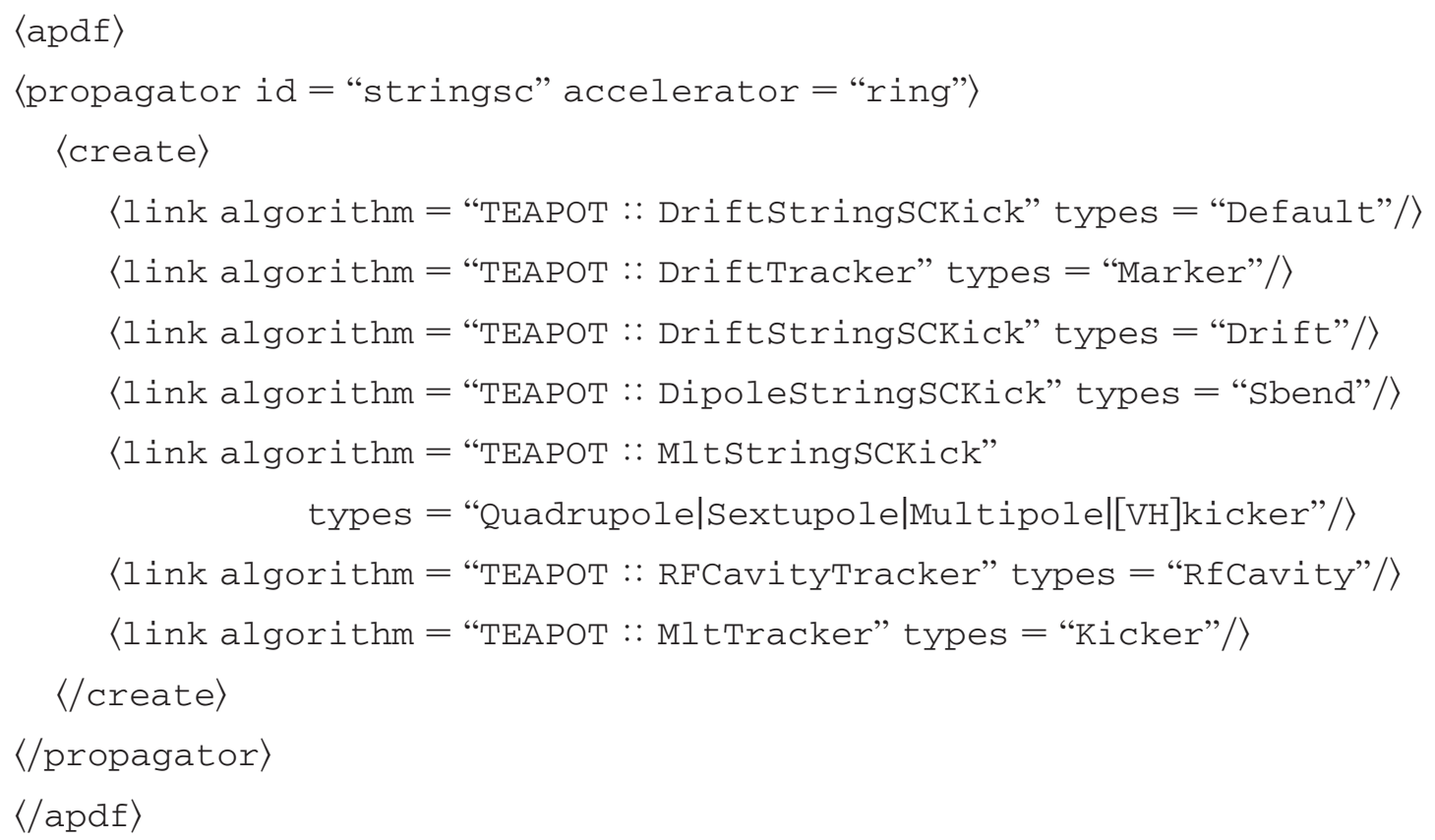

This file implements the UAL architectural principle according to which the lattice description file contains absolutely no specification as to what algorithms are to be employed in evolving particle or bunch properties through the lattice. The linkages of algorithms to lattice element types are completely specified in this ".apdf" file. (More complicated linkages, such as different algorithms for differently named elements of the same type, are also possible, but are not needed here.) Simply by changing the algorithm linkages, the space charge tracking can be turned on or off. All parameters governing the space charge are given on the command line (or, somewhat less flexibly, in the program itself) of the "main.cc" program, which provides the UAL "command language" mechanism.

The lattice itself (with all its parameters) is described in the so-called ".sxf" (standard exchange format) file. This file is most easily (and completely mechanically) produced as a fully instantiated output from MAD-X [35], starting from a standard MAD input lattice description.
All results in this paper have been obtained using a laptop computer, with $N_{p}$, the number of particles tracked, being of order 1000 (mainly $N_{p}=800$ or $N_{p}=1600$ ). Such a small number of particles might be expected to cause unacceptably noisy results. In fact the various plots in this paper are relatively smooth.

For reduced granularity, one would prefer to track perhaps 10 times as many particles. With the computation time scaling as $N_{p}^{2}$, this would make the computation unacceptably slow. One contemplates, therefore, using parallel processing to reduce the computation time by a factor almost proportional to the number of parallel computers, $N_{c}$, especially if one wishes to track bunches for one or more full turns around a circular ring.

For the string space charge method, the computation time is dominated by the $N_{p}^{2}$ calculations of the space charge force on every particle by every other particle. Propagating the particles from one space charge kick location to the next is relatively quick. A natural division of 
the labor is to divide the $N_{p}$ particles into groups each containing $N_{p} / N_{c}$ particles, with one group allotted to each computer, which calculates all space charge deflections for its own group of particles, and propagates them to the next space charge location. The parallelization overhead comes from broadcasting all $6 N_{p}$ particle coordinates to all $N_{c}$ computers each time a space charge kick location is encountered. (At the moment the code uses only the three position coordinates, but an eventually more accurate calculation would depend also on the slope coordinates.)

[1] H. Braun, Report No. CERN/PS 2000-030 (AE), CLICNote 441, 2000.

[2] H. Braun et al., Phys. Rev. Lett. 84, 658 (2000).

[3] H. Braun et al., Phys. Rev. ST Accel. Beams 3, 124402 (2000).

[4] M. Chanudet-Cayla, CLIC Note 429, PS/RF Note 2000006, 2000.

[5] L. Groening et al., in Proceedings of the Particle Accelerator Conference, Chicago, IL, 2001 (IEEE, New York, 2001), http://groening.home.cern/groening/csr_00. htm.

[6] H. Braun et al., in Proceedings of the Particle Accelerator Conference, Chicago, IL, 2001, Ref. [5], p. 164.

[7] R. Talman, Phys. Rev. Lett. 56, 1429 (1986).

[8] M. Bassetti, Report No. CERN/LEP-TH/86-13, 1986; M. Bassetti and D. Brandt, Report No. CERN/LEP-TH/86-04, 1986.

[9] R. Talman, Phys. Rev. ST Accel. Beams 7, 100701 (2004).

[10] N. Malitsky and R. Talman, in Proceedings of the 9th European Particle Accelerator Conference, Lucerne, 2004 (EPS-AG, Lucerne, 2004).

[11] R. Talman, Accelerator X-Ray Sources (Wiley-VCH, Weinheim, 2006), Chap. 13.

[12] A. Kabel et al., Nucl. Instrum. Methods Phys. Res., Sect. A 455, 185 (2000).

[13] M. Borland, Argonne National Laboratory Report No. LS287, 2000.
[14] Ya. S. Derbenev et al., TESLA-FEL, 95-05, 1995.

[15] M. Dohlus and T. Limberg, in Proceedings of the 21st Particle Accelerator Conference, Knoxville, 2005 (IEEE, Piscataway, NJ, 2005).

[16] F. Stulle et al., Phys. Rev. ST Accel. Beams 10, 031001 (2007).

[17] R. Li and Y. Derbenev, Jefferson Lab Report No. LJABTN-02-054, 2003.

[18] G. Geloni et al., arXiv:physics/0310133v1.

[19] B. Beutner et al., in Proceedings of FEL Conference, BESSY, Berlin, Germany, 2006, MOPPH009.

[20] E. Saldin et al., Nucl. Instrum. Methods Phys. Res., Sect. A 398, 373 (1997).

[21] B. Carlsten, Phys. Rev. E 54, 838 (1996).

[22] R. Hajima and E. Ikeno, Nucl. Instrum. Methods Phys. Res., Sect. A 429, 315 (1999).

[23] J. Jackson, Classical Electrodynamics (John Wiley, New York, 1999), 3rd ed.

[24] E. Saldin et al., Nucl. Instrum. Methods Phys. Res., Sect. A 398, 373 (1997).

[25] G. Geloni et al., arXiv:physis/0205001v1.

[26] R. Talman and N. Malitsky, Cornell Report No. CBN 0711, 2007.

[27] J. Schwinger, Report No. LBNL-39088/CBP, Note 179, 1996.

[28] F. Chautard, Ph.D. thesis, University of Paris, 1986.

[29] M. Dohlus and T. Limberg, in Proceedings of the 2004 FEL Conference, pp. 18-21, MOCOS05, available at http://www.JACoW.org.

[30] Coherent Synchrotron Radiation, CSR Workshop, Berlin 2002, http://www.desy.de/csr.

[31] L. Giannessi, Phys. Rev. ST Accel. Beams 6, 114802 (2003).

[32] P. Emma, CSR Benchmark Test-Case Results, CSR Workshop, Berlin 2002.

[33] A. Piwinski, The Touschek Effect in Strong Focusing Storage Rings, ISSN 0418-9833 [DESY Report 98-179, 1998].

[34] UAL website http://www.agsrhichome.bnl.gov/UAL/.

[35] MAD-X website http://mad.web.cern.ch/mad/. 